\title{
Burn-in e Política de Manutenção
}

\author{
Perseverando da indade Garcia finho
}

INSTITUTO DE MATEMÁTICA E ESTATÍSTICA

DA

UNIVERSIDADE DE SÃO PAULO

PARA OBTENÇÃO DO GRAU DE MESTRE

EM

ESTATÍSTICA

\author{
Área de Concentração : Probabilidade \\ Orientador : Prof. Dr. Vanderlei da Costa Bueno \\ O autor recebeu apoio financeiro do \\ Governo do Estado do Amazonas \\ - São Paulo, dezembro de 2003 -
}




\section{BURN-IN E POLÍTICA DE MANUTENÇÃO}

Este exemplar corresponde à redação final da dissertação devidamente corrigida e apresentada por Perseverando da Trindade Garcia Filho e aprovada pela Comissão Julgadora.

São Paulo, 17 de fevereiro de 2004.

Banca Examinadora:

Prof. Dr. Vanderlei da Costa Bueno (Orientador) - IME/USP

Prof. Dr. Luis Gustavo Esteves - IME/USP

Prof. Dr. João Batista Camargo Junior - Escola Politécnica/USP 


\section{Agradecimentos}

Agradeço primeiramente a DEUS, por ter me dado força e saúde para realizar este trabalho.

Ao prof. Vanderlei, pela orientação, paciência e compreensão com minhas dificuldades.

Aos meus pais, Tereza e Perseverando ${ }^{\dagger}$, meus irmãos e a todos os familiares.

A Edijane Garcia, uma grande companheira, pelo carinho e apoio nas horas mais duras.

A Diana Galvis, por me presentear com sua valiosa amizade.

Aos professores Celso Rômulo, José Cardoso e Rosana Parente.

Aos amigos, Shirley \& Telamon (meu irmão espiritual), Claudete, Graça, Dra. Fátima, ao mestre Afonso Lins e família, Alberto (assessor de futebol), Delhi, Diana \& Gustavo (brother de apê), Elmo, Iran e Willian (trio inseparável), Jaqueline \& Juvêncio (Vozão/10), Mariela, Michele \& Horácio, Prof. Luiz Gustavo (corinthiano feliz), Elier (grande cubano e parceiro), Rita \& Osnel, Sandra \& Cristian, Santos, Waldemar (guerreiro), Maria do Carmo, Ulisses e David (BioInfo), que tanto colaboraram para que eu alcançasse o meu objetivo.

Aos amigos da Cândido Mariano, do Banik e do futebol, sempre torcendo por mim.

A uma amostra representativa de colegas, da biblioteca (Nádia, Célia, Lu, Max, Chico, Alê, Elzinha, Zé, Sílvia,...), da portaria (Marcos, Xavier), do cafezinho, da Xerox, Adriana, Alberto, Ângela, Daniela \& Marcos, Domingos, Felipe (tranquilito), José (Zamorano), Gisela, Joelmir, Ricardo, Kleber, Lia, Lourdes, Márcio, Mauricio, Nathalia, Nubia, Paola, Patrícia, Paulo, Paulo Tadeu, Pinho (por nos atender sempre com muita educação), Silvina, Hugo (hugol), Jorge (patas), Rogério, Val, Rejane, Carlos (Col), Rodrigo Catedral, Thales, Michel, Caio, Giane e a todos que de uma forma ou de outra participaram desta minha jornada. 


\section{Sumário}

1 Introdução 2

2 Procedimento de Burn-in 4

3 Burn-in e Política de Manutenção por Idade 16

4 Burn-in e Política de Manutenção por Bloco 26

5 Modelos Gerais: Comentários 36

5.1 Burn-in com reparos mínimos e política de manutenção por blocos com reparos

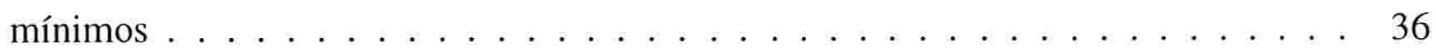

5.2 Procedimento de burn-in condicionado a tipos de falhas do objeto . . . . . . . . 41

$\begin{array}{lll}\text { I Conclusão } & 51\end{array}$

II Apêndice $\quad 52$

III Bibliografia $\quad 55$ 


\section{Resumo}

O burn-in é um método utilizado para prevenir falhas precoces de um objeto (sistema ou componente), melhorando sua qualidade.

Em geral, um procedimento de burn-in continua até assegurarmos uma confiabilidade máxima para um tempo de funcionamento prefixado ou minimizarmos o número médio de falhas durante um período prefixado de funcionamento.

Nesta dissertação analisamos as políticas de manutenção por idade e por bloco de objetos reparáveis que tenham sobrevivido a um teste de burn-in. Consideramos reparos completos (no caso de política de manutenção por idade) e reparos mínimos (no caso de política de manutenção por bloco) e minimizamos o custo de uma tal política sobre tais objetos colocados em operação. Em ambas as situações, minimizamos uma estrutura de custo total que inclui o custo do procedimento de burn-in e o custo da política de manutenção.

\section{Abstract}

Burn-in is a preventive method to eliminate initial failure of an object (system or component) improving its quality.

In general we apply a burn-in procedure to, either, allow a maximum realibility for a prefixed mission type or a minimum mean number of failure during a period of a given mission.

In this dissertation we analyse age and block maintenance policies of burned-in objects. In the case of age maintenance policy, we consider complete repairs and in the case o block maintenance policy, we consider minimal repairs. On either situations we minimize a total cost structure including the cost of the burn-in procedure and the cost of the maintenance policy. 


\section{Capítulo 1}

\section{Introdução}

Burn-in é um procedimento usado em engenharia para eliminar itens defeituosos em uma linha de produção. Uma linha de produção consiste usualmente na fabricação de componentes os quais são estruturalmente organizados formando sistemas de engenharia. Podemos considerar, por exemplo, sistemas que são circuitos eletrônicos compostos por vários tipos de chips. Os sistemas, assim como os componentes, operam por um período de tempo e falham.

Dentro de uma linha de produção existem, normalmente, componentes defeituosos com tempo de vida menores que o desejado e componentes perfeitos, "não defeituosos", com tempo de vida grande o suficiente para nossos propósitos.

Para assegurar que somente os componentes perfeitos sejam colocados no mercado o fabricante submeterá, por um período de tempo, todos os componentes, ou uma amostra representativa destes, a testes que simulem situações típicas, e até mesmo mais severas, do uso do componente no mercado. Estes tipos de testes também podem ser realizados em sistemas com o objetivo de determinar os que são defeituosos ou os que apresentam defeitos estruturais.

Na prática, a ocorrência dessas falhas, tanto no aspecto econômico quanto no operacional, podem ser desastrosa e de consequencias imprevisíveis, de forma que políticas de manutenção com o objetivo de reduzir tais ocorrências são benéficas. 
Adotada uma política de manutenção uma estrutura de custo total é estabelecida e um $T$ ótimo é determinado de forma que o custo total seja minimizado. Duas políticas comuns nas aplicações são a política de manutenção por idade e a política de manutenção por bloco. Sob a suposição de que a distribuição do objeto tem taxa de falha crescente, Barlow e Proschan (1965) provou que, para essas políticas, uma política ótima existe, podendo ser finita.

A política de manutenção ótima, certamente, depende da função de distribuição do objeto utilizado na operação. Portanto, é natural levar em consideração o procedimento conjunto de burn-in e de política de manutenção preventiva e, assim, novos modelos são exigidos para descrever o custo total incorrido pelos dois procedimentos induzidos conjuntamente. Determinada a estrutura do custo total, procuramos um tempo ótimo de burn-in, e uma política de manutenção ótima, tal que a função de custo total seja minimizada.

Nesta dissertação, baseada em Mi (1994), o nosso propósito é analisar políticas de manutenção em objetos que sobreviveram ao procedimento de Burn-in.

Aqui assumimos que a distribuição $F$ do objeto original, antes do burn-in, tem taxa de falha na forma bathtub. Sob essa suposição, as conclusões obtidas são de que tempo ótimo de burn-in ocorre no período de mortalidade infantil e o tempo ótimo de manutenção, somado ao tempo ótimo de burn-in, ocorre no período de desgaste.

No Capítulo 2, dissertamos de uma maneira geral, sobre o procedimento de burn-in critérios baseados na performance dos objetos ou nos custos operacionais. No Capítulo 3 analisamos a Política de Manutenção por Idade e no Capítulo 4 a Política de Manutenção por Blocos quando consideramos conjuntamente o procedimento de burn-in. No Capítulo 5 comentamos sobre os resultados recentes no desenvolvimento da teoria. 


\section{Capítulo 2}

\section{Procedimento de Burn-in}

Para o propósito desta dissertação, usaremos o termo burn-in de uma maneira geral: como uma pré-operação de uso do objeto que envolve condições normais ou estressantes; que envolve partes ou toda a população de objetos; que não é limitado à eliminação de objetos defeituosos.

Como um exemplo, que aparece em Block e Savits (1997), Rawicz (1986) considerou lâmpadas de 30 watts fabricadas pela Pacific Lamp Corporation (Vancover, Canadá), as quais são projetadas para uma durabilidade de 5.000 horas de funcionamento contínuo a uma voltagem de 120V.

As lâmpadas seriam instaladas em um out-door de maneira que seria difícil e de custo elevado realizar substituições no caso de falha. Era conhecido que uma pequena porcentagem das lâmpadas falhavam antes das 5.000 horas e, portanto, seria benéfico se essa subpopulação de lâmpadas fosse identificada antes de ser instalada no out-door.

Adotou-se um procedimento de testar todas as lâmpadas a uma voltagem de $240 \mathrm{~V}$, por um período de tempo, o que causou a falha das lâmpadas defeituosas rapidamente. As lâmpadas perfeitas que sobreviveram a este período, sobreviveriam às 5.000 horas de funcionamento contínuo.

Frequentemente, o procedimento de burn-in deteriora os objetos, contudo, neste experimento particular, as lâmpadas que sobreviveram ao teste tiveram aumento do seu tempo de vida.

Um outro exemplo do AT\&T Realibility Manual (Klinger, Nakada e Menendez, 1990) aparece 
em Block e Savits (1997). Considera-se um sistema eletrônico de switching (interruptores, chaves). Imediatamente depois de sua fabricação o sistema é colocado em operação a uma temperatura de $25^{\circ} \mathrm{C}$ por 12 horas durante o qual um volume de chamadas é testado, mais precisamente, 1.000 chamadas são simuladas através de 5 a 8 módulos do switch. Em adição, o sistema é sujeito a uma temperatura alta de $50^{\circ} \mathrm{C}$, o que poderia ocorrer em cada switch quando o ar-condicionado falhasse. A finalidade da primeira parte do procedimento é encontrar e eliminar falhas precoces do sistema. A segunda parte simula um caso extremo acelerando o processo de envelhecimento de maneira que sistemas menos confiáveis sejam eliminados.

Muitas das idéias tradicionais concernentes ao burn-in são discutidas no livro de Jensen e Petersen (1982). Uma das contribuições importantes do livro é popularizar a idéia que objetos para os quais o procedimento de burn-in é recomendado possuem tempos de vida que podem ser modelados como misturas de funções de distribuições de probabilidade.

Especificamente, os componentes são originados de subpopulações de defeituosos e perfeitos e seus tempos de vida podem ser modelados como misturas de distribuições dos tempos de vida dos itens dessas subpopulações. Assume-se também que os tempos de vida dos sistemas tenham origem da mesma forma e que a subpopulação de defeituosos é caracterizada pela mortalidade infantil, isto é, o objeto tem taxa de falha inicial alta . Esta subpopulação origina-se, parcialmente, dos defeitos introduzidos pelo processo de fabricação.

Consideramos um objeto com tempo de vida aleatório $X$ definido em um espaço de probabilidade $(\Omega, \Im, P)$ com função de distribuição $F$ absolutamente contínua, com $F(t)<1, \forall t \geq 0$.

Sejam $f$ a função densidade de probabilidade associada a $F$ e $r$ a taxa de falha de $X$, definida por:

$$
r(t)=\frac{f(t)}{\bar{F}(t)}=\lim _{d t \rightarrow 0} \frac{P(X \in(t, t+d t] \mid X \geq t)}{d t}
$$

em que $\bar{F}(t)=1-F(t)$ é a função de sobrevivência de $X$.

A taxa de falha, $r(t)$, é a propensão a falha de um objeto com idade t, isto é, a probabilidade do objeto falhar instantaneamente no intervalo $(t, t+d t]$ condicionado a que ele sobreviva à idade $t$. 
De uma forma geral poderíamos perguntar:

Para quais funções de distribuições úteis na modelagem dos tempos de vida de objetos o procedimento de burn-in é efetivo?

Em princípio é razoável que desprezemos as distribuições com taxas de falhas crescentes pois neste caso os objetos tornam-se propensos à falhas com a idade e o procedimento de burn-in deterioraria o tempo de vida residual. Portanto as distribuições com taxas de falha crescente ( IFR) não são, geralmente, candidatas para um procedimento de burn-in.

Para que o procedimento de burn-in seja efetivo o tempo de vida do objeto deveria ter uma taxa de falha inicial alta e então, uma taxa de falha decrescente com o tempo (uma propensão de falha menor depois de um tempo inicial). Desde que os objetos que sobrevivam ao procedimento possuem a mesma taxa de falha do que o objeto original truncada à esquerda, o procedimento de burn-in elimina parte do tempo inicial na qual o objeto teria uma alta propensão a falha.

A classe de tempos de vida tendo taxa de falha na forma bathtub tem esta propriedade. Para este tipo de distribuição a taxa de falha inicial é alta (mortalidade infantil), então decresce para uma constante, aproximadamente (tempo de vida útil), e, em seguida cresce com a idade avançada (período de desgaste). Como sugerido entre parentêses, essas distribuições são adequadas para modelar o tempo de vida dos seres humanos e outros tempos de vida biológicos. Certos tempos de vida de objetos eletrônicos também podem ser aproximados por tais distribuições.

Neste trabalho assumimos que a taxa de falha $r(t)$ tem a forma Bathtub.

\section{Definição 1:}

Uma função a valores não negativos, $r(t)$, tem a forma bathtub se existe $0 \leq t_{1} \leq t_{2}<\infty$ tal que $r(t)$ é:

$$
\left\{\begin{array}{llr}
\text { estritamente decrescente, } & \text { se } \quad 0 \leq t \leq t_{1} ; \\
\text { constante, } & \text { se } t_{1} \leq t \leq t_{2} e \\
\text { estritamente crescente, } & \text { se } \quad t_{2} \leq t<\infty .
\end{array}\right.
$$




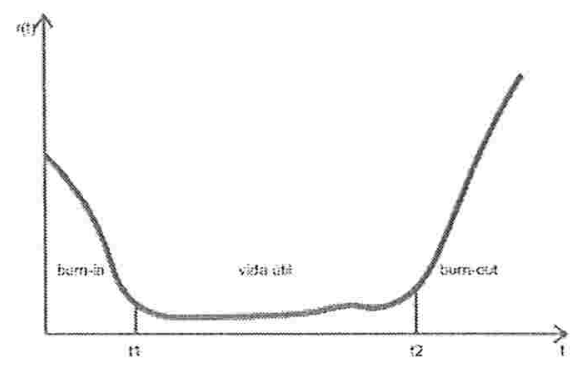

Figura 2.1: Função Bathtub

Os instantes $t_{1}$ e $t_{2}$ são chamados de pontos de mudança (troca) de $r(t)$.

Interpretamos os intervalos de tempo:

$\left[0, t_{1}\right]$ como o período de mortalidade infantil,

$\left[t_{1}, t_{2}\right]$ como o período de vida útil em que $r(t)$ é aproximadamente constante e

$\left[t_{2}, \infty\right)$ é chamado período fora de uso, ou desgaste.

Acredita-se que muitos produtos, particularmente os eletrônicos, tais como circuitos integrados, possuem taxa de falha na forma bathtub. Esse ponto de vista tem suporte em muitas experiências e coleções de dados feitas por pesquisadores em muitas indústrias.

Por simplicidade, na definição de função de taxa de falha consideramos $t_{2}<\infty$.

Existem razões pelas quais muitos objetos tem a taxa de falha na forma bathtub. Como descrito por Jensen e Petersen (1982), muitas populações originadas da produção industrial são heterogêneas em um número pequeno de subpopulações diferentes. Embora os membros dessas subpopulações não tenham taxa de falha na forma bathtub, a amostragem destas produzem misturas cujas distribuições têm, frequentemente, taxa de falha na forma bathtub. Para um exemplo simples assuma, que existam duas subpopulações de objetos, cada uma modelada por tempo de vida com distribuição exponencial. Uma subpopulação com tempo de vida médio pequeno e outra subpopulação com tempo de vida médio grande. O tempo de vida de objetos amostrados desta população são misturas cujas as taxas de falha associada tem a forma bathtub. Uma explicação 
intuitiva para este resultado é que, inicialmente, a alta taxa de falha da subpopulação (com média menor) é predominante até que esta subpopulação se extinga. Em seguida, a baixa taxa de falha da outra subpopulação (com média maior) se evidencia de forma que a taxa de falha decresce de níveis altos para níveis baixos.

A mistura de duas exponenciais mencionada acima produz um caso especial de taxa de falha na forma bathtub, onde a fase em que o objeto esta no período de desgaste (idade avançada) não é evidente. Modelos desse tipo são considerados suficientes para aproximar os tempos de vida de certos componentes eletrônicos, os quais em geral, tornam-se obsoletos antes de uma idade avançada.

Misturas de distribuições mais complexas podem produzir distribuições com taxas de falhas que tem, tipicamente, a forma bathtub.

Quando decidimos por um procedimento de burn-in, devemos considerar algum critério básico segundo o qual passamos a determinar o tempo ótimo de burn-in. Alguns exemplos de critérios que envolvem a "perfomance" do objeto são:

A) Seja $\tau$ (fixado) o tempo desejado de operação de um objeto com função de sobrevivência $\bar{F}$. Encontrar b que maximize $\frac{\bar{F}(b+\tau)}{\bar{F}(b)}$, isto é, encontrar b tal que, condicionado a que o objeto sobreviveu ao instante b, a probabilidade de sobreviver o tempo desejado de operação $\tau$ seja máxima.

B) Seja X o tempo de vida de um objeto. Encontrar o tempo de burn-in b que maximize o tempo de vida médio residual $E[X-b \mid X>b]$.

C) Seja $\left\{N_{b}(t), t \geq 0\right\}$ um processo de renovação onde a distribuição entre as ocorrências tem função de sobrevivência $P(X>t+b \mid X>b)=\frac{\bar{F}(b+\tau)}{\bar{F}(b)}$, de objetos com função de distribuição (original) $\mathrm{F}$ e que sobreviveram ao procedimento de burn-in de b unidades de tempo. Para um 
tempo desejado de operação $\tau$, encontrar b que minimize o número médio de componentes que sobreviveram ao procedimento de burn-in e que falham durante o tempo de operação desejado $\tau$, $E\left[N_{b}(\tau)\right]$

D) Em C, uma unidade que falha durante o tempo de operação desejado é substituída por outra unidade semelhante (que sobreviveu ao procedimento de burn-in). Se, ao invés de substituirmos a unidade, realizarmos um reparo mínimo, o número total de reparos mínimos é um processo de Poisson não homogêneo com média $-\ln \left[\frac{\bar{F}(b+\tau)}{\bar{F}(b)}\right]$. Assim, se desejamos minimizar o número esperado de reparos mínimos no intervalo $[0, \tau]$, é suficiente maximizar a quantidade $\frac{\bar{F}(b+\tau)}{\bar{F}(b)}$, que é justamente o critério A.

Embora critérios baseados na performance do objeto sejam úteis as situações mais realistas envolvem estruturas de custos. Várias estruturas de custos tem sido propostas e, em geral, temos interesse em encontrar o tempo de burn-in b que minimize tais funções. Alguns exemplos são:

E) Um objeto com tempo de vida X é submetido a um procedimento de burn-in com tempo de duração b. O objeto que não sobrevive ao tempo b tem um custo $C_{o}$. Se ele sobrevive ao tempo b, mas não sobrevive ao tempo adicional desejado $\tau$ um custo $C>C_{o}$ ocorre. Se o objeto sobrevive a $\tau+b$ temos um ganho de $\mathrm{k}$ unidades. Consequentemente, se $\mathrm{F}$ é a função de distribuição do objeto, o custo esperado como função de b é

$$
C(b)=C_{o} F(b)+C[F(b+\tau)-F(b)]-k \bar{F}(b+\tau) .
$$

F) Se ao invés de um período desejado de operação, depois do tempo de burn-in b, considerarmos um ganho proporcional k, o custo esperado é

$$
C(b)=C_{o} F(b)-k \frac{\int_{b}^{\infty} \bar{F}(t) d t}{\bar{F}(b)} .
$$


G) Um objeto novo é submetido a um procedimento de burn-in com tempo fixado b. Se o objeto falha antes do tempo b, é reparado a um custo $C_{s}>0$ e submetido novamente ao procedimento de burn-in, e, assim, sucessivamente. Aqui, assumimos que o reparo é completo, isto é, o objeto reparado é tão bom quanto um novo. Se o objeto sobrevive ao tempo b de burn-in, é colocado em operação. O custo para este procedimento é proporcional ao tempo total do burn-in com constante de proporcionalidade $C_{o}>0$. O custo médio do procedimento é obtido da seguinte maneira:

Sejam $F$ a função de distribuição de um objeto novo e $\left\{X_{i}, i \geq 1\right\}$ uma sequência de variáveis aleatórias independentes e identicamente distribuídas com distribuição $F$. Seja $\eta-1$ a variável aleatória que conta o número de reparos até que o objeto sobreviva ao tempo de burn-in fixado, b. Assim o custo para este procedimento é dado por:

$$
h(b)=C_{o}\left(\sum_{i=1}^{\eta-1} X_{i}+b\right)+C_{s}(\eta-1) .
$$

Note que a variável aleatória $X_{\eta}-b$ tem função de sobrevivência

$$
P\left(X_{\eta}-b>s\right)=P\left(X_{\eta}>b+s\right)=\left\{\begin{array}{lll}
\frac{\bar{F}(b+s)}{\bar{F}(b)} & , \text { se } & s \geq 0 \\
1 & , \text { se } & s<0
\end{array}\right.
$$

e que a variável aleatória $\eta$ tem distribuição geométrica dada por:

$P(\eta=k)=\bar{F}(b) F^{k-1}(b)$, com média $\frac{1}{\bar{F}(b)}$.

Como, para todo instante $\mathrm{n}$, podemos determinar se $\eta$ ocorreu, ou não, observando $X_{1}, X_{2}, \ldots, X_{n}$, aplicando a identidade de Wald temos que,

$$
\begin{aligned}
E\left(\sum_{i=1}^{\eta-1} X_{i}\right)= & E(\eta) E\left(X_{1}\right)-E\left(X_{\eta}\right)=\frac{1}{\bar{F}(b)} \int_{0}^{\infty} \bar{F}(t) d t-\left(\int_{b}^{\infty} \frac{\bar{F}(t)}{\bar{F}(b)} d t+b\right) \\
& =\frac{1}{\bar{F}(b)} \int_{0}^{b} F(t) d t+\frac{1}{\bar{F}(b)} \int_{b}^{\infty} \bar{F}(t) d t-\int_{b}^{\infty} \frac{\bar{F}(t)}{\bar{F}(b)} d t-b
\end{aligned}
$$




$$
=\frac{1}{\bar{F}(b)} \int_{0}^{b} \bar{F}(t) d t-b .
$$

Adicionando $b$ aos dois membros da equação, temos

$$
E\left(\sum_{i=1}^{\eta-1} X_{i}+b\right)=\int_{0}^{b} \frac{\bar{F}(t)}{\bar{F}(b)} d t
$$

Podemos agora calcular o custo esperado como

$$
\begin{aligned}
k(b)= & E(h(b))=E\left[C_{o}\left(\sum_{i=1}^{\eta-1} X_{i}+b\right)+C_{s}(\eta-1)\right]= \\
& \left.=E\left[C_{o}\left(\sum_{i=1}^{\eta-1} X_{i}+b\right)\right)\right]+E\left(C_{s}(\eta-1),\right.
\end{aligned}
$$

e assim,

$$
k(b)=C_{o} \int_{0}^{b} \frac{\bar{F}(t)}{\bar{F}(b)} d t+\frac{C_{s} F(b)}{\bar{F}(b)} .
$$

A função de custo pode incluir também custos aditivos de operação.

H) Depois que um objeto sobrevive ao tempo de burn-in b, um custo $C$ ocorre se o objeto não sobrevive ao tempo (adicional) de operação desejado $\tau$ e um ganho k se êle sobrevive a este tempo:

$$
C(b)=k(b)+C \frac{F(b+\tau)-F(b)}{\bar{F}(b)}-k \frac{\bar{F}(b+\tau)}{\bar{F}(b)}
$$

onde $k(b)$ é como definido em G.

I) Se, ao invés de um tempo de operação $\tau$, temos um ganho proporcional ao tempo médio residual, então:

$$
C(b)=k(b)-k \frac{\int_{b}^{\infty} \bar{F}(t) d t}{\bar{F}(b)} .
$$


Os objetos podem ser reparáveis ou não. Se um objeto não reparável falha, é substituído por outro objeto com tempo de vida independente e identicamente distribuído ao tempo de vida do objeto substituído.

Se o objeto é reparável, consideramos dois tipos de reparos: No primeiro, no chamado reparo completo, um objeto que falha é reparado e fica tão bom quanto a um novo, com um tempo de vida independente e identicamente distribuído ao tempo de vida original. O segundo tipo de reparo é o reparo mínimo, isto é, o reparo reconstitui a taxa de falha do objeto como era imediatamente anterior à falha.

\section{Definição 2:}

Sob uma Política de Manutenção por Idade o objeto é substituído na sua falha ou na idade T (uma constante fixa), a qual ocorrer primeiro.

J) Se o tempo de vida do objeto é $\mathrm{X}$, com função de distribuição $\mathrm{F}$ absolutamente contínua, o número de substituições em uma política de manutenção por idade constitui um processo de renovação em que um ciclo, o intervalo entre as renovações, tem comprimento aleatório $\min (X, T)$. Assim o tempo médio de um ciclo é

$$
\mu=E[\min (X, T)]=\int_{0}^{T}(1-F(x)) d x .
$$

Se $C_{f}$ é o custo da falha do objeto em operação e $C_{a}$ é o custo de uma substituição na idade T, o custo do i-ésimo ciclo aleatório $Y_{i}$ é dado por

$$
Y_{i}=\left\{\begin{array}{l}
C_{f} F(T) \text { se } X_{i}<T \\
C_{a}(1-F(T)) \text { se } X_{i} \geq T
\end{array}\right.
$$

e, portanto,

$$
E\left(Y_{i}\right)=C_{f} F(T)+C_{a}(1-F(T))
$$


Aplicando a teoria dos Processos de Renovação, o custo médio por unidade de tempo, assintótico, é dado por

$$
C(T)=\frac{E\left(Y_{i}\right)}{\mu}=\frac{C_{f} F(T)+C_{a}(1-F(T))}{\int_{0}^{T}(1-F(x)) d x} .
$$

Se o objeto em uso já sobreviveu ao procedimento de burn-in a função de sobrevivência do tempo de vida residual $X_{b}=(X \mid X>b)$ é $\bar{F}_{X_{b}}(s)=P\left(X_{b}>s\right)=$

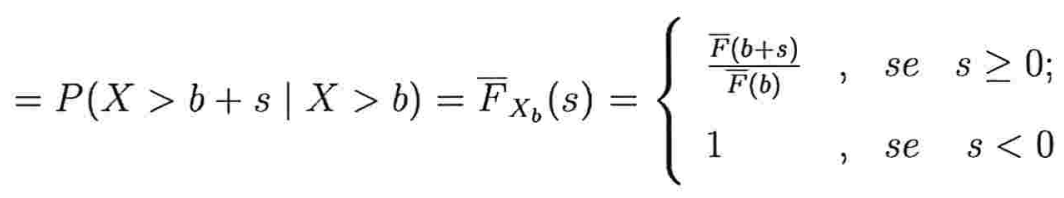

e o custo médio por unidade de tempo, assintótico, passa a ser

$$
C(b, T)=\frac{\left.C_{f} F_{X_{b}}(T)+C_{a} \bar{F}_{X_{b}}(T)\right)}{\int_{0}^{T} \bar{F}_{X_{b}}(t) d t} .
$$

Se desejarmos incorporar em nossa função o custo devido ao procedimento de burn-in, como em $(\mathrm{G})$, teremos

$$
C(b, T)=\frac{\left.C_{f} F_{X_{b}}(T)+C_{a} \bar{F}_{X_{b}}(T)\right)+k(b)}{\int_{0}^{T} \bar{F}_{X_{b}}(t) d t} .
$$

Uma outra política comum é a Política de Manutenção por Blocos.

\section{Definição 3:}

Sob uma Política de Manutenção por Bloco, o objeto é substituído na falha ou nos tempos kT, $\mathrm{k}=1,2, \ldots . .$, onde $\mathrm{T}$ é um número fixado.

K) Se considerarmos uma política de manutenção por blocos na qual realizamos reparos mínimos nas falhas do objeto em operação a um custo $C_{m}$ e substituições planejadas a cada $\mathrm{T}$ unidade de tempo, obteremos ciclos de comprimentos $\mathrm{T}$ com custos $Y_{i} \mathrm{e}$

$$
E\left(Y_{i}\right)=C_{\tau}+C_{m} E\left[N_{m}(T)\right]
$$


onde

$C_{\tau}$ é o custo da reposição planejada;

$C_{m}$ é o custo do reparo mínimo;

$N_{m}(T)$ é o número de reparos mínimos em $[0, T]$.

Prova-se que $E\left[N_{m}(T)\right]=\int_{0}^{T} r(s) d s$, ver [1], onde $r(s)$ é a taxa de falha do objeto.

Assim, o custo médio por unidade de tempo, assintótico, é

$$
C(T)=\frac{C_{m} \int_{0}^{T} r(s) d s+C_{\tau}}{T}
$$

Se consideramos objetos que sobreviveram ao procedimento de burn-in, a função de custo torna-se

$$
C(b, T)=\frac{C_{m} \int_{b}^{b+T} r(s) d s+C_{\tau}}{T}
$$

Se desejamos incorporar à função o custo devido ao procedimento de burn-in e se, como em G, o custo médio de burn-in é $k(b)$, o correspondente custo médio assintótico por unidade de tempo é

$$
\frac{1}{T}\left(-C_{s}+\frac{C_{s}+C_{o} \int_{0}^{b} \bar{F}(x) d x}{\bar{F}(b)}\right)
$$

e a função de custo total torna-se

$$
C(b, T)=\frac{1}{T}\left(-C_{s}+\frac{C_{s}+C_{o} \int_{0}^{b} \bar{F}(x) d x}{\bar{F}(b)}\right)+\frac{C_{\tau}+C_{m} \int_{b}^{b+T} r(t) d t}{T}
$$

Adotada uma política de manutenção uma estrutura de custo total é estabelecida e um $T$ ótimo (denotado por $T^{*}$ ) é determinado de forma que o custo total seja minimizado. $T^{*}$ é chamado de política de manutenção ótima. Sob a suposição de que $F$ tem taxa de falha crescente (IFR), Barlow e Proschan (1965) provou que, para uma política de manutenção por idade ou para uma política de manutenção por blocos com reparos mínimos nas falhas, uma política ótima existe (podendo ser infinita). 
A política de manutenção ótima, certamente, depende da função de distribuição do objeto utilizado na operação. Portanto, é natural levar em consideração o procedimento conjunto de burn-in e política de manutenção preventiva e, assim, novos modelos são exigidos para descrever o custo total incorrido pelos dois procedimentos simultaneamente. Determinada a estrutura do custo total, como em Mi (1994), procuramos um tempo ótimo de burn-in, $b^{*}$, e uma política de manutenção ótima, $T^{*}$, tal que a função de custo total seja minimizada.

Aqui assumimos que a distribuição $F$ do objeto original, antes do burn-in, tem taxa de falha $r(t)$ na forma bathtub. Sob essa suposição, as conclusões obtidas são de que $b^{*}$ (tempo ótimo de burn-in) ocorre no período de mortalidade infantil da $r(t)$ e a soma $b^{*}+T^{*}$ ocorre no período de desgaste. 


\section{Capítulo 3}

\section{Burn-in e Política de Manutenção por Idade}

Inicialmente consideramos a política de manutenção por idade como descrita em Barlow e Proschan (1965). Seja $C_{f}$ o custo de uma falha do objeto em operação e $C_{a}$, satisfazendo $0<C_{a}<C_{f}$, o custo de uma substituição de um objeto em operação na idade $T>0$. Se usarmos somente os objetos que tenham sobrevivido ao tempo de burn-in b, o custo médio por unidade de tempo,assintótico, $C(b, T)$, como em J (Capítulo 2), do procedimento de manutenção é dado por (Barlow e Proschan,1965):

$$
C(b, T)=\frac{C_{f} F_{X_{b}}(T)+C_{a} \bar{F}_{X_{b}}(T)+k(b)}{\int_{0}^{T} \bar{F}_{X_{b}}(t) d t}
$$

em que $\bar{F}_{X_{b}}(T)$ é a função de sobrevivência do tempo de vida residual, isto é, $\bar{F}_{X_{b}}(x)=\frac{\bar{F}(b+x)}{\bar{F}(b)}$, $k(b)=E(h(b))$, onde $h(b)$ é definida em G do Capítulo 2. Assim,

$$
C(b, T)=\frac{C_{f}[\bar{F}(b)-\bar{F}(b+T)]+C_{a} \bar{F}(b+T)+k(b) \bar{F}(b)}{\int_{0}^{T} \bar{F}(b+t) d t} .
$$

Os resultados com respeito ao tempo ótimo de burn-in $b^{*}$ e a política de manutenção por idade 
ótima $T^{*}$, os quais satisfazem

$$
C\left(b^{*}, T^{*}\right)=\min _{b>0 ; T>0} C(b, T)
$$

foram desenvolvidos por Jie Mi (1994) e são dados no seguinte teorema:

\section{Teorema 3.1:}

Se a taxa de falha $r(t)$ tem a forma bathtub e é diferenciável então, sob uma política de manutenção por idade com reparo completo, o tempo ótimo de burn-in $b^{*}$ e a correspondente política de manutenção ótima $T^{*}=T^{*}\left(b^{*}\right)$ satisfazem $0 \leq b^{*} \leq t_{1}$ e $b^{*}+T^{*}=b^{*}+T^{*}\left(b^{*}\right)>t_{2}$, onde $T^{*}(b)$ é igual a $\infty$ ou é a única solução da equação abaixo, dependendo, respectivamente, se a equação tem ou não solução.

$$
r(b+T) \int_{0}^{T} \frac{\bar{F}(b+t)}{\bar{F}(b)} d t+\frac{\bar{F}(b+T)}{\bar{F}(b)}=\frac{C_{f}+k(b)}{C_{f}-C_{a}} .
$$

\section{Prova:}

Note que o valor esperado $k(b)=E(h(b))=C_{o} \int_{0}^{b} \frac{\bar{F}(t)}{\bar{F}(b)} d t+\frac{C_{s} F(b)}{\bar{F}(b)}$ é uma função estritamente crescente em b e converge para $\infty$ quando $b \uparrow \infty$. Com o objetivo de minimizar

$$
C(b, T)=\frac{C_{f}[\bar{F}(b)-\bar{F}(b+T)]+C_{a} \bar{F}(b+T)+k(b) \bar{F}(b)}{\int_{0}^{T} \bar{F}(b+t) d t}
$$

como função de T, calculamos a primeira derivada parcial, $\frac{\partial C}{\partial T}$,

$$
\frac{\left[C_{f} f(b+T)-C_{a} f(b+T)\right] \int_{0}^{T} \bar{F}(b+t) d t-\left\{C_{f}[\bar{F}(b)-\bar{F}(b+T)]+C_{a} \bar{F}(b+T)+k(b) \bar{F}(b)\right\} \bar{F}(b+T)}{\left[\int_{0}^{T} \bar{F}(b+t) d t\right]^{2}}
$$

Assim, $\frac{\partial C}{\partial T}=0$ se, e somente se,

$$
\left(C_{f}-C_{a}\right) f(b+T) \int_{0}^{T} \bar{F}(b+t) d t-\left\{\left(C_{a}-C_{f}\right) \bar{F}(b+T)+\left(C_{f}+k(b)\right) \bar{F}(b)\right\} \bar{F}(b+T)=0,
$$


o que é equivalente às expressões abaixo:

$\frac{\left(C_{f}-C_{a}\right) f(b+T) \int_{0}^{T} \bar{F}(b+t) d t}{\bar{F}(b+T)}=-\left(C_{f}-C_{a}\right) \bar{F}(b+T)+\left(C_{f}+k(b)\right) \bar{F}(b) \Leftrightarrow$

$\frac{f(b+T)}{\bar{F}(b+T)} \int_{0}^{T} \bar{F}(b+t) d t\left(C_{f}-C_{a}\right)=-\left(C_{f}-C_{a}\right) \bar{F}(b+T)+\left(C_{f}+k(b)\right) \bar{F}(b) \Leftrightarrow$

$\frac{f(b+T)}{\bar{F}(b+T)} \int_{0}^{T} \bar{F}(b+t) d t\left(C_{f}-C_{a}\right)+\left(C_{f}-C_{a}\right) \bar{F}(b+T)=\left(C_{f}+k(b)\right) \bar{F}(b) \Leftrightarrow$

$r(b+T) \frac{\left(C_{f}-C_{a}\right) \int_{0}^{T} \bar{F}(b+t) d t}{\bar{F}(b)}+\frac{\left(C_{f}-C_{a}\right) \bar{F}(b+T)}{\bar{F}(b)}=C_{f}+k(b) \Leftrightarrow$

$r(b+T) \frac{\int_{0}^{T} \bar{F}(b+t) d t}{\bar{F}(b)}+\frac{\bar{F}(b+T)}{\bar{F}(b)}=\frac{C_{f}+k(b)}{C_{f}-C_{a}}$.

Concluimos que, $\frac{\partial C}{\partial T}=0$ se, e somente se,

$$
r(b+T) \frac{\int_{0}^{T} \bar{F}(b+t) d t}{\bar{F}(b)}+\frac{\bar{F}(b+T)}{\bar{F}(b)}=\frac{C_{f}+k(b)}{C_{f}-C_{a}} .
$$

Deduzimos também que $\frac{\partial C}{\partial T}<0$ se, e somente se,

$$
r(b+T) \frac{\int_{0}^{T} \bar{F}(b+t) d t}{\bar{F}(b)}+\frac{\bar{F}(b+T)}{\bar{F}(b)}<\frac{C_{f}+k(b)}{C_{f}-C_{a}} .
$$

Para um b, fixado definimos, convenientemente, a função auxiliar

$$
\psi_{b}(T)=r(b+T) \frac{\int_{0}^{T} \bar{F}(b+t) d t}{\bar{F}(b)}+\frac{\bar{F}(b+T)}{\bar{F}(b)} .
$$

Note que

$\psi_{b}(0)=1$ e $\psi_{b}(\infty)=r(\infty) \mu(b)$.

Derivando $\psi_{b}(T)$ com relação a $T$ temos, 


$$
\begin{aligned}
\psi_{b}^{\prime}(T) & =r^{\prime}(b+T) \frac{\int_{0}^{T} \bar{F}(b+t) d t}{\bar{F}(b)}+r(b+T) \frac{\bar{F}(b+T)}{\bar{F}(b)}-\frac{f(b+T)}{\bar{F}(b)}= \\
& =r^{\prime}(b+T) \frac{\int_{0}^{T} \bar{F}(b+t) d t}{\bar{F}(b)}+r(b+T) \frac{\bar{F}(b+T)}{\bar{F}(b)}-\frac{f(b+T)}{\bar{F}(b+T)} \frac{\bar{F}(b+T)}{\bar{F}(b)}= \\
& =r^{\prime}(b+T) \frac{\int_{0}^{T} \bar{F}(b+t) d t}{\bar{F}(b)} .
\end{aligned}
$$

Como, por hipótese, a taxa de falha $r(t)$ tem a forma bathtub concluimos que, em relação a $\mathrm{T}$, $\psi_{b}(T)$ é

$$
\left\{\begin{array}{lrr}
\text { estritamente decrescente, } & \text { se } & b+T \leq t_{1} \\
\text { constante, } & \text { se } & t_{1} \leq b+T \leq t_{2} \text { e } \\
\text { estritamente crescente, } & \text { se } & b+T \geq t_{2} .
\end{array}\right.
$$

Desde que o custo esperado k(b) é positivo, temos

$k(b)>-C_{a} \Rightarrow C_{f}+k(b)>C_{f}-C_{a} \Rightarrow \frac{C_{f}+k(b)}{C_{f}-C_{a}}>1$.

Além disso

$\psi_{b}(0)=1$ e $\psi_{b}(T)$ é decrescente em $T$ se $b+T \leq t_{2}$. Portanto, $\frac{\partial C}{\partial T}<0$ para $b+T \leq t_{2}$ e para que tenhamos

$$
C\left(b, T^{*}(b)\right)=\min _{T>0} C(b, T(b))
$$

com $b \geq 0$ e arbitrário, é necessário que

$$
b+T^{*}>t_{2}
$$

Por outro lado se para algum $b, T^{*}(b)=\infty$, da expressão (3.1), temos

$$
C(b, \infty)=\frac{C_{f}+k(b)}{\mu(b)}
$$

Para continuar definimos, convenientemente, os subconjuntos de $\Re^{+}$ 
$B_{1}=\left\{b \geq 0: T^{*}(b)<\infty\right\} \mathrm{e}$

$B_{2}=\left\{b \geq 0: T^{*}(b)=\infty\right\}$.

Podemos obsevar que

$B_{1}=\left\{b>0: \psi_{b}(\infty)>\frac{C_{f}+k(b)}{C_{f}-C_{a}}\right\}=\left\{b>0: \mu(b)>\frac{1}{r(\infty)} \frac{C_{f}+k(b)}{C_{f}-C_{a}}\right\}$.

Consequentemente $B_{2}$, o complementar de $B_{1}$, é dado por

$B_{2}=\left\{b>0: \mu(b) \leq \frac{1}{r(\infty)} \frac{C_{f}+k(b)}{C_{f}-C_{a}}\right\}$.

Observamos também que:

- O tempo de vida residual de um objeto com idade $b, b>0$, tem função de sobrevivência

$$
\bar{F}_{X_{b}}(s)= \begin{cases}\frac{\bar{F}(b+s)}{\bar{F}(b)}, & \text { se } s \geq 0 \\ 1 & , \text { se } s<0 .\end{cases}
$$

- Se o objeto tem tempo de vida médio finito, a esperança matemática do tempo de vida residual é dado por

$$
\mu(b)=E[X-b \mid X>b]=\int_{b}^{\infty} \frac{\bar{F}(x)}{\bar{F}(b)} d x .
$$

- Suponha que $\mu(b)$ admita um máximo em $\tilde{b}$. Mi (1991) provou que $0 \leq \tilde{b} \leq t_{1}$ e que $\mu(b)$

$$
\left\{\begin{array}{rrr}
\text { estritamente crescente, } & \text { se } 0 \leq b \leq \tilde{b} \text { e } \\
\text { estritamente decrescente, } & \text { se } & b>\tilde{b} .
\end{array}\right.
$$

Desde que o custo esperado $k(b)$ é crescente em b, temos que $\frac{C_{f}+k(b)}{\mu(b)}$ é crescente em $b>\tilde{b}$ (pois $\mu(b)$ é estritamente decrescente em $b>\tilde{b})$.

Para prosseguir, consideramos dois casos:

Caso 1) $B_{1}=\varnothing$

Neste caso temos que $T^{*}(b)=\infty$ e

$C\left(b, T^{*}(b)\right)=C(b, \infty)=\frac{C_{f}+k(b)}{\mu(b)}>C\left(t_{1}, \infty\right)$ para $b>t_{1}$.

Consequentemente $b^{*} \leq t_{1}$ e $T^{*}(b)=\infty$. 
Caso 2) $B_{1} \neq \varnothing$

Como $\mu(\infty)=\lim _{b \rightarrow \infty} \mu(b)=\lim _{b \rightarrow \infty} \frac{1}{\bar{F}(b)} \int_{b}^{\infty} \bar{F}(t) d t=\lim _{b \rightarrow \infty} \frac{\bar{F}(b)}{f(b)}=\frac{1}{r(\infty)}$ e $\lim _{b \rightarrow \infty} k(b)=\infty, B_{1}$ é limitado. Como $\mu(b)$ e $k(b)$ são funções contínuas e a imagem inversa de um intervalo aberto através de uma função contínua é um aberto concluímos que $B_{1}$ é um intervalo aberto e limitado. Como $T^{*}(b)$ satisfaz a equação (3.4) temos

$$
\frac{\int_{0}^{T^{*}(b)} \bar{F}(b+t) d t}{\bar{F}(b)}=\frac{\frac{C_{f}+k(b)}{C_{f}-C_{a}}-\frac{\bar{F}\left(b+T^{*}(b)\right)}{\bar{F}(b)}}{r\left(b+T^{*}(b)\right)} .
$$

Substituindo na expressão (3.1), temos as igualdades

$$
\begin{aligned}
C\left(b, T^{*}(b)\right) & =\frac{C_{f}\left[\bar{F}(b)-\bar{F}\left(b+T^{*}(b)\right)\right]+C_{a} \bar{F}\left(b+T^{*}(b)\right)+k(b) \bar{F}(b)}{\int_{0}^{T^{*}(b)} \bar{F}(b+t) d t} \\
& =r\left(b+T^{*}(b)\right) \frac{C_{f}\left[\bar{F}(b)-\bar{F}\left(b+T^{*}(b)\right)\right]+C_{a} \bar{F}\left(b+T^{*}(b)\right)+k(b) \bar{F}(b)}{\frac{\left(C_{f}+k(b)\right) \bar{F}(b)}{\left(C_{f}-C_{a}\right) \bar{F}(b)}-\frac{\left(C_{f}-C_{a}\right) \bar{F}\left(b+T^{*}(b)\right)}{\left(C_{f}-C_{a}\right) \bar{F}(b)}}
\end{aligned}
$$

isto é,

$$
C\left(b, T^{*}(b)\right)=r\left(b+T^{*}(b)\right)\left(C_{f}-C_{a}\right)
$$

o que reduz o problema original a minimizar $r\left(b+T^{*}(b)\right)$ sobre $b \geq 0$. Consideramos dois subcasos Subcaso 1) $\sup B_{1}=\beta \leq t_{1}$

Como $B_{1}$ é aberto e limitado temos que $[\beta, \infty) \subseteq B_{2}$. Desde que $C(b, \infty)=\frac{C_{f}+k(b)}{\mu(b)}$ é crescente em $b$ no intervalo $\left(t_{1}, \infty\right)$, temos

$$
\min _{b \in[\beta, \infty)} C\left(b, T^{*}(b)\right)=\min _{b \in[\beta, \infty)} C(b, \infty)
$$




$$
\begin{aligned}
& =\min _{b \in[\beta, \infty)} \frac{C_{f}+k(b)}{\mu(b)} \\
& =\min _{b \in\left[\beta, t_{1}\right]} \frac{C_{f}+k(b)}{\mu(b)} \\
& =\min _{b \in\left[\beta, t_{1}\right]} C\left(b, T^{*}(b)\right) .
\end{aligned}
$$

Assim,

$$
\begin{aligned}
\min C\left(b, T^{*}(b)\right) & =\min \left\{\min _{b \in[0, \beta)} C\left(b, T^{*}(b)\right) ; \min _{b \in[\beta, \infty)} C\left(b, T^{*}(b)\right)\right\} \\
& =\min _{b \in\left[0, t_{1}\right]} C\left(b, T^{*}(b)\right) \Rightarrow b^{*} \leq t_{1} .
\end{aligned}
$$

Subcaso 2) $\sup B_{1}=\beta>t_{1}$

Seja b qualquer número em $[\tilde{b}, \beta]$. Como $\mu(b)$ é decrescente em $(\tilde{b}, \infty)$ e $k(b)$ é crescente,

$$
\mu(b)>\mu(\beta)=\frac{1}{r(\infty)} \frac{C_{f}+k(\beta)}{C_{f}-C_{a}}>\frac{1}{r(\infty)} \frac{C_{f}+k(b)}{C_{f}-C_{a}}
$$

Portanto $b \in B_{1}$, que implica que $[\tilde{b}, \beta) \subseteq B_{1}$ e consequentemente $\left[t_{1}, \beta\right) \subseteq B_{1}$.

Se $b \in\left[t_{1}, \beta\right)$ a expressão (3.4) vale para $T^{*}(b)$. Assim,

$$
r\left(b+T^{*}(b)\right) \int_{0}^{T^{*}(b)} \bar{F}(b+t) d t+\bar{F}\left(b+T^{*}(b)\right)=\frac{C_{f}+k(b)}{C_{f}-C_{a}} \bar{F}(b) .
$$

Derivando a expressão acima como função de b, temos:

$$
\begin{aligned}
& r^{\prime}\left(b+T^{*}(b)\right)\left(1+T^{* *}(b)\right) \int_{0}^{T^{*}(b)} \bar{F}(b+t) d t+r\left(b+T^{*}(b)\right)\left\{-\int_{0}^{T^{*}(b)} f(b+t) d t+T^{* *}(b) \bar{F}\left(b+T^{*}(b)\right)\right\}- \\
& f\left(b+T^{*}(b)\right)\left(1+T^{*}(b)\right)=\frac{k^{\prime}(b) \bar{F}(b)}{C_{f}-C_{a}}-f(b) \frac{C_{f}+k(b)}{C_{f}-C_{a}}
\end{aligned}
$$


o que é equivalente, para todo $b \in\left[t_{1}, \beta\right)$, a

$r^{\prime}\left(b+T^{*}(b)\right)\left(1+T^{* *}(b)\right) \int_{0}^{T^{*}(b)} \bar{F}(b+t) d t=\bar{F}(b)\left[r\left(b+T^{*}(b)\right)+\frac{k^{\prime}(b)}{C_{f}-C_{a}}-r(b) \frac{C_{f}+k(b)}{C_{f}-C_{a}}\right](3$

Provaremos que o segundo termo da igualdade é positivo.

Escrevendo convenientemente

$$
r\left(b+T^{*}(b)\right)+\frac{k^{\prime}(b)}{C_{f}-C_{a}}-r(b) \frac{C_{f}+k(b)}{C_{f}-C_{a}}=\frac{N(b)}{\left(C_{f}-C_{a}\right) \int_{0}^{T^{*}(b)} \bar{F}(b+t) d t}
$$

concluimos que é suficiente provar que $N(b)>0$. Como de (3.12) temos

$$
r\left(b+T^{*}(b)\right)=\frac{\frac{C_{f}+k(b)}{C_{f}-C_{a}} \bar{F}(b)-\bar{F}\left(b+T^{*}(b)\right)}{\int_{0}^{T^{*}(b)} \bar{F}(b+t) d t}
$$

segue, de (3.14), que

$$
\begin{array}{r}
\frac{\left(C_{f}+k(b)\right) \bar{F}(b)-\left(C_{f}-C_{a}\right) \bar{F}\left(b+T^{*}(b)\right)}{\left(C_{f}-C_{a}\right) \int_{0}^{T^{*}(b)} \bar{F}(b+t) d t}+\frac{k^{\prime}(b)}{\left(C_{f}-C_{a}\right)}-\frac{r(b)\left(C_{f}+k(b)\right)}{\left(C_{f}-C_{a}\right)}= \\
=\frac{N(b)}{\left(C_{f}-C_{a}\right) \int_{0}^{T^{*}(b)} \bar{F}(b+t) d t}
\end{array}
$$

e, portanto,

$$
\begin{aligned}
N(b)= & C_{f}\left[\bar{F}(b)-\bar{F}\left(b+T^{*}(b)\right)\right]+C_{a} \bar{F}\left(b+T^{*}(b)\right)+k(b) \bar{F}(b)+k^{\prime}(b) \int_{0}^{T^{*}(b)} \bar{F}(b+t) d t- \\
& -\left(C_{f}+k(b)\right) r(b) \int_{0}^{T^{*}(b)} \bar{F}(b+t) d t .
\end{aligned}
$$

Como, por hipótese, $r(t)$ tem a forma bathtub, para todo $b \in\left[t_{1}, \beta\right)$ temos

$$
r(b) \int_{0}^{T^{*}(b)} \bar{F}(b+t) d t<\int_{0}^{T^{*}(b)} r(b+t) \bar{F}(b+t) d t=\bar{F}(b)-\bar{F}\left(b+T^{*}(b)\right)
$$


o que implica que

$$
C_{f}\left[\bar{F}(b)-\bar{F}\left(b+T^{*}(b)\right)\right]+C_{a} \bar{F}\left(b+T^{*}(b)\right)>C_{f} r(b) \int_{0}^{T^{*}(b)} \bar{F}(b+t) d t
$$

Em adição,

$$
k(b) \bar{F}(b)+k^{\prime}(b) \int_{0}^{T^{*}(b)} \bar{F}(b+t) d t>k(b)\left[\bar{F}(b)-\bar{F}\left(b+T^{*}(b)\right)\right]>k(b) r(b) \int_{0}^{T^{*}(b)} \bar{F}(b+t) d t .(3.17)
$$

A primeira desigualdade é verdadeira, pois $k(b)>0$ e $k^{\prime}(b)>0$ e a segunda desigualdade segue de (3.15).

Se observarmos as equações (3.16) e (3.17) notamos que elas são parcelas de $N(b)$ e dessa forma podemos concluir que $N(b)>0, \forall b \in\left[t_{1}, \beta\right)$.

Como

$$
r^{\prime}\left(b+T^{\prime *}(b)\right)\left(1+T^{* *}(b)\right) \int_{0}^{T^{*}(b)} \bar{F}(b+t) d t>0 \Rightarrow\left(1+T^{* *}(b)\right)>0, \forall b \in\left[t_{1}, \beta\right)
$$

$\Rightarrow b+T^{*}(b)$ é estritamente crescente em $b \in\left[t_{1}, \beta\right)$ e consequentemente

$$
\min _{b \in B_{1}} C\left(b, T^{*}(b)\right)=\min _{b \in B_{1}}\left(C_{f}-C_{a}\right) r\left(b+T^{*}(b)\right)
$$

só pode ser obtido em $\left[0, t_{1}\right]$, desde que $\left(b+T^{*}(b)\right)>t_{2}$ e $r(t)$ é crescente em $t \geq t_{2}$. Por outro lado,

$$
\min _{b \geq \beta} C\left(b, T^{*}(b)\right)=\min _{b \geq \beta} C(b, \infty)=\min _{b \geq \beta} \frac{C_{f}+k(b)}{\mu(b)}=\frac{C_{f}+k(\beta)}{\mu(\beta)},
$$

e concluimos que

$\beta \in B_{2}$ e $\mu(\beta)=\frac{1}{r(\infty)} \frac{C_{f}+k(\beta)}{C_{f}-C_{a}}$, uma vez que, $\mu(b)$ e $k(b)$ são funções contínuas. Assim,

$$
\min _{b \geq \beta} C\left(b, T^{*}(b)\right)=\frac{C_{f}+k(\beta)}{\mu(\beta)}=\left(C_{f}-C_{a}\right) r(\infty)>\left(C_{f}-C_{a}\right) r\left(t_{1}+T^{*}\left(t_{1}\right)\right) \geq \min _{b \in B_{1}} C\left(b, T^{*}(b)\right)
$$


o que produz imediatamente $b^{*} \leq t_{1}$ o qual satisfaz

$$
C\left(b^{*}, T^{*}(b)\right)=\min _{b \geq 0} C\left(b, T^{*}(b)\right)=\min _{b \geq 0 ; T>0} C(b, T) .
$$

\section{Exemplo 3.1:}

Como um exemplo de aplicação do teorema considere um objeto com tempo de vida $X$, função de distribuição $F$ e função de sobrevivência $\bar{F}(t)=e^{1-e^{(\lambda t)^{\alpha}}}$. Consequentemente a taxa de falha, $r(t)=\alpha \lambda^{\alpha} t^{\alpha-1} e^{(\lambda t)^{\alpha}}$, tem a forma bathtub, onde $t_{1}=t_{2}=\frac{1}{\lambda}$ é o ponto de mudança, para $\alpha=0.5$. O tempo ótimo de burn-in $b^{*}$ e a política de manutenção ótima $T^{*}$ devem satisfazer a equação:

$$
\begin{gathered}
0.5 \lambda^{0.5}(b+T)^{0.5-1} e^{(\lambda(b+T))^{0.5}} \int_{0}^{T} \frac{e^{1-e^{(\lambda(b+t))^{0.5}}} d t}{e^{1-e^{(\lambda b)^{0.5}}}}+\frac{e^{1-e^{(\lambda(b+T))^{0.5}}}}{e^{1-e^{(\lambda b)^{0.5}}}}=\frac{c_{f}+k(b)}{c_{f}-c_{a}} \\
\text { onde } k(b)=c_{o} \int_{0}^{b} \frac{e^{1-e^{(\lambda t)^{0.5}}} d t}{e^{1-e^{(\lambda b)^{0.5}}}+\frac{c_{s}\left(1-e^{1-e^{(\lambda b)^{0.5}}}\right)}{e^{1-e^{(\lambda b)^{0.5}}}}}
\end{gathered}
$$

e são tais que $b^{*} \leq \frac{1}{\lambda}$ e $b^{*}+T^{*}>\frac{1}{\lambda}$.

Para verificar tal propriedade, uma simulação é feita assumindo $\lambda=3$ e para cada valor de $b_{i}$, $\mathrm{i}=1, \ldots, 20$, é realizada 100 interações para $\mathrm{T}$ no domínio, $b \leq \frac{1}{3}$ e $T \leq \frac{1}{3}-b$.

Neste domínio não encontramos nenhuma solução da equação, o que reforça os resultados do teorema, pois qualquer solução, se existir, estará no domínio $b \leq \frac{1}{3}$ e $T \geq \frac{1}{3}-b$. 


\section{Capítulo 4}

\section{Burn-in e Política de Manutenção por Bloco}

Neste Capítulo consideramos uma política de manutenção por bloco com reparos mínimos nas falhas do objeto. Mais precisamente, fixamos um $T>0$, substituímos o objeto nos tempos $k T, k=1,2,3, \ldots$ e realizamos reparos mínimos nas eventuais falhas intermediárias. O reparo mínimo reconstitui a taxa de falha do objeto como era imediatamente anterior a falha.

Sejam $C_{m}>0$ o custo do reparo mínimo, $C_{r}>0$ o custo da reposição, $N_{r}(t)$ o número de reposições no intervalo $[0, t]$ e $N_{m}(t)$ o número de reparos mínimos em $[0, t]$. O custo médio por unidade de tempo, assintótico, da política de manutenção de objetos que sobreviveram ao procedimento de burn-in é

$$
\frac{C_{r}+C_{m} \int_{0}^{T} r_{X_{b}}(x) d x}{T}
$$

onde $r_{X_{b}}(x)$ é a taxa de falha da função de distribuição $F_{X_{b}}$ definida por $F_{X_{b}}(s)=1-\frac{\bar{F}(b+s)}{\bar{F}(b)}$, a função de distribuição de um objeto que sobreviveu ao tempo de burn-in b.

Claramente, das equivalências

$\int_{0}^{s} r_{X_{b}}(x) d x=\left[-\log \bar{F}_{X_{b}}(s)\right] \Longleftrightarrow$

$\frac{\partial}{\partial s} \int_{0}^{s} r_{X_{b}}(x) d x=\frac{\partial}{\partial s}\left[-\log \frac{\bar{F}(b+s)}{\bar{F}(b)}\right] \Longleftrightarrow$ 
$r_{X_{b}}(s)=\frac{f(b+s)}{F(b+s)} \Longleftrightarrow$

$r_{X_{b}}(s)=r(b+s)$,

segue que $r_{X_{b}}(x)=r(b+x)$

Substituindo em (4.1) temos

$$
\frac{C_{r}+C_{m} \int_{0}^{T} r(b+s) d s}{T} .
$$

Tomando $x=b+s$, podemos reescrever a equação na forma

$$
\frac{C_{r}+C_{m} \int_{b}^{b+T} r(x) d x}{T} .
$$

Como o custo do procedimento de cada objeto é $h(b)$ e o número de reposições em $[0, t]$ durante a operação é $N_{r}(t)=\left\lfloor\frac{t}{T}\right\rfloor(\lfloor x\rfloor$ é o maior número inteiro menor ou igual a x), o custo total associado ao procedimento de burn-in é $\left(N_{r}(t)+1\right) h(b)$. Desde que $N_{r}(t)$ não é aleatório, o custo médio por unidade de tempo, assintótico, do procedimento de burn-in é

$$
\begin{aligned}
\lim _{t \rightarrow \infty} \frac{E\left(N_{r}(t)+1\right)(h(b))}{t} & =\lim _{t \rightarrow \infty} \frac{\left(N_{r}(t)+1\right) E(h(b))}{t} \\
& =\lim _{t \rightarrow \infty} \frac{N_{r}(t)+1}{t}\left[\frac{C_{o} \int_{0}^{b} \bar{F}(t) d t}{\bar{F}(b)}+\frac{C_{s} F(b)}{\bar{F}(b)}\right] \\
& =\lim _{t \rightarrow \infty} \frac{\left\lfloor\frac{t}{\bar{T}}\right\rfloor+1}{t}\left[\frac{C_{o} \int_{0}^{b} \bar{F}(t) d t}{\bar{F}(b)}+\frac{C_{s}(1-\bar{F}(b))}{\bar{F}(b)}\right] \\
& =\frac{1}{T}\left[\frac{C_{o} \int_{0}^{b} \bar{F}(t) d t}{\bar{F}(b)}+\frac{C_{s}}{\bar{F}(b)}-\frac{C_{s}(\bar{F}(b)}{\bar{F}(b)}\right] \\
& =\frac{1}{T}\left[\frac{C_{s}+C_{o} \int_{0}^{b} \bar{F}(t) d t}{\bar{F}(b)}-C_{s}\right]
\end{aligned}
$$


A função de custo total é a combinação das equações (4.2) e (4.3):

$$
C(b, T)=\frac{1}{T}\left[-C_{s}+\frac{C_{s}+C_{o} \int_{0}^{b} \bar{F}(t) d t}{\bar{F}(b)}\right]+\frac{C_{r}+C_{m} \int_{b}^{b+T} r(t) d t}{T}
$$

Nosso objetivo é selecionar $b^{*}$ e $T^{*}$ tais que

$$
C\left(b^{*}, T^{*}\right)=\min _{0 \leq b<\infty ; 0<T \leq \infty} C(b, T)
$$

As propriedades de $b^{*}$ e da política ótima de manutenção por blocos $T^{*}$, semelhantes ao afirmado no teorema 3.1, são

\section{Teorema 4.1:}

Se a taxa de falha $r(t)$ tem a forma bathtub é diferenciável e se o conjunto

$B_{1}=\left\{b \geq 0: \int_{b}^{\infty}[r(\infty)-r(t)] d t>\frac{1}{C_{m} \bar{F}(b)}\left[\left(C_{r}-C_{s}\right) \bar{F}(b)+\left(C_{s}+C_{o} \int_{0}^{b} \bar{F}(t) d t\right)\right]\right\}$

é diferente do vazio, então $0 \leq b^{*} \leq t_{1}$ e $\infty>b^{*}+T^{*}\left(b^{*}\right)>t_{2}$, onde $T^{*}(b)$ é a solução única da equação

$$
\operatorname{Tr}(b+T)-\int_{b}^{b+T} r(t) d t=\frac{1}{C_{m} \bar{F}(b)}\left[\left(C_{r}-C_{s}\right) \bar{F}(b)+\left(C_{s}+C_{o} \int_{0}^{b} \bar{F}(t) d t\right)\right]
$$

e $b^{*}$ é o tempo de burn-in ótimo, isto é,

$C\left(b^{*}, T^{*}\left(b^{*}\right)\right)<C\left(b, T^{*}(b)\right)<C(b, T)$,

$\forall b \neq b^{*}$ e $\forall T \neq T^{*}(b), \forall b \geq 0$.

Se o conjunto acima é vazio, então para qualquer $b \geq 0$ temos

$T^{*}(b)=\infty, C\left(b, T^{*}(b)\right)=C_{m} r(\infty)$ e $T^{*}(b)$ satisfaz

$C\left(b, T^{*}(b)\right)<C(b, T), \forall T \neq T^{*}(b)$. 


\section{Prova:}

Segue, de (4.4), que para todo $0<T \leq \infty$ temos

$C(\infty, T)=\lim _{b \rightarrow \infty} C(b, T)=\infty$. Assumimos também que $b^{*} \neq \infty$.

Para qualquer b fixado, $b \in[0, \infty)$, a derivada parcial de $C(b, T)$ em relação a $\mathrm{T}$ é igual a

$$
\begin{aligned}
\frac{\partial C}{\partial T} & =-\frac{1}{T^{2}}\left[-C_{s}+\frac{C_{s}+C_{o} \int_{0}^{b} \bar{F}(t) d t}{\bar{F}(b)}\right]+\frac{1}{T^{2}}\left[C_{m} \operatorname{Tr}(b+T)-\left(C_{r}+C_{m} \int_{b}^{b+T} r(t) d t\right)\right]= \\
& =-\frac{1}{T^{2}}\left[-C_{s}+\frac{C_{s}+C_{o} \int_{0}^{b} \bar{F}(t) d t}{\bar{F}(b)}\right]+\frac{1}{T^{2}}\left[C_{m} \operatorname{Tr}(b+T)-C_{m} \int_{b}^{b+T} r(t) d t-C_{r}\right] .
\end{aligned}
$$

Definindo convenientemente

$$
\psi_{b}(T)=\operatorname{Tr}(b+T)-\int_{b}^{b+T} r(t) d t
$$

temos

$$
\begin{aligned}
& \frac{\partial C}{\partial T}=-\frac{1}{T^{2}}\left[-C_{s}+\frac{\left.C_{s}+C_{o} \int_{0}^{b} \bar{F}(t) d t\right]}{\bar{F}(b)}\right]+\frac{1}{T^{2}}\left[C_{m} \psi_{b}(T)-C_{r}\right] \\
= & -\frac{1}{T^{2}}\left[\frac{C_{s}+C_{o} \int_{0}^{b} \bar{F}(t) d t-C_{s} \bar{F}(b)-C_{m} \psi_{b}(T) \bar{F}(b)+C_{r} \bar{F}(b)}{\bar{F}(b)}\right] \\
= & -\frac{1}{T^{2}}\left[\frac{\left(C_{r}-C_{s}\right) \bar{F}(b)+C_{s}+C_{o} \int_{0}^{b} \bar{F}(t) d t-C_{m} \psi_{b}(T) \bar{F}(b)}{\bar{F}(b)}\right] \\
= & -\frac{C_{m}}{T^{2}}\left[\frac{\left(C_{r}-C_{s}\right) \bar{F}(b)+C_{s}+C_{o} \int_{0}^{b} \bar{F}(t) d t}{C_{m} \bar{F}(b)}-\frac{C_{m} \psi_{b}(T) \bar{F}(b)}{C_{m} \bar{F}(b)}\right] \\
= & \frac{C_{m}}{T^{2}}\left\{\psi_{b}(T)-\frac{1}{C_{m} \bar{F}(b)}\left[\left(C_{r}-C_{s}\right) \bar{F}(b)+\left(C_{s}+C_{o} \int_{0}^{b} \bar{F}(t) d t\right)\right]\right\} .
\end{aligned}
$$


Assim, $\frac{\partial C}{\partial T}=0$ se, e somente se,

$$
\psi_{b}(T)=\frac{1}{C_{m} \bar{F}(b)}\left[\left(C_{r}-C_{s}\right) \bar{F}(b)+\left(C_{s}+C_{o} \int_{0}^{b} \bar{F}(t) d t\right)\right]
$$

e $\frac{\partial C}{\partial T}<0$ se, e somente se

$$
\psi_{b}(T)<\frac{1}{C_{m} \bar{F}(b)}\left[\left(C_{r}-C_{s}\right) \bar{F}(b)+\left(C_{s}+C_{o} \int_{0}^{b} \bar{F}(t) d t\right)\right] .
$$

Pelo teorema da convergência monótona, de (4.6) temos

$$
\psi_{b}(\infty)=\lim _{T \rightarrow \infty} \psi_{b}(T)=\lim _{T \rightarrow \infty} \int_{b}^{b+T}[r(b+T)-r(t)] d t=\int_{b}^{\infty}[r(\infty)-r(t)] d t
$$

Definimos, de maneira conveniente, os subconjuntos

$$
\begin{aligned}
& B_{1}=\left\{b \geq 0: \psi_{b}(\infty)=\int_{b}^{\infty}[r(\infty)-r(t)] d t>\frac{1}{C_{m} \bar{F}(b)}\left[\left(C_{r}-C_{s}\right) \bar{F}(b)+\left(C_{s}+C_{o} \int_{0}^{b} \bar{F}(t) d t\right)\right]\right\} \\
& \mathrm{e} \\
& B_{2}=[0, \infty) \backslash B_{1} .
\end{aligned}
$$

Consideramos 3 casos:

Caso 1) $B_{1}=[0, \infty)$ e $B_{2}=\emptyset$

Isto acontece se, e somente se,

$\psi_{b}(\infty)=\int_{b}^{\infty}[r(\infty)-r(t)] d t=\infty$, para pelo menos um $b \geq 0$, pois a expressão à direita em (4.8) converge para $\infty$ quando $b \rightarrow \infty$. Em particular, isto ocorre quando $r(\infty)=\infty$ e $r(0)<\infty$.

Como $\psi_{b}(T)=\operatorname{Tr}(b+T)-\int_{b}^{b+T} r(t) d t$, segue que 
$\left.\psi_{b}^{\prime}(T)=r(b+T)\right]+T r^{\prime}(b+T)-r(b+T)=T r^{\prime}(b+T)$

e consequentemente $\psi_{b}(T)$, como função de T é

$$
\left\{\begin{array}{lrr}
\text { estritamente decrescente, } & \text { se } & 0 \leq T \leq t_{1}-b ; \\
\text { constante, } & \text { se } & t_{1}-b \leq T \leq t_{2}-b e \\
\text { estritamente crescente, } & \text { se } & t_{2}-b \leq T .
\end{array}\right.
$$

Desde que $\psi_{b}(0)=0, \psi_{b}(\infty)=\infty, C_{r}, C_{m}$ e $C_{o}$ são positivos, a equação

$$
\psi_{b}(T)=\frac{C_{r}-C_{s}}{C_{m}}+\frac{C_{s}+C_{o} \int_{0}^{b} \bar{F}(t) d t}{C_{m} \bar{F}(b)}
$$

tem solução única, a qual denotamos por $T^{*}(b)$. Além disso, como $\psi_{b}$ é monótona e $\psi_{b}(0)=0$, temos que $\psi_{b}(T) \leq 0, \forall T \in\left[0, t_{2}-b\right]$. Isto mostra que a única solução de (4.11), $T^{*}(b)$, satisfaz $T^{*}(b)>t_{2}-b$ para um dado $b>0$. Assim mostramos que

$$
t_{2}-b<T^{*}(b)<\infty
$$

Como $T^{*}(b)$ satisfaz a expressão (4.11) temos

$$
T^{*}(b) r\left(b+T^{*}(b)\right)-\int_{b}^{b+T^{*}(b)} r(t) d t=\frac{C_{r}-C_{s}}{C_{m}}+\frac{C_{s}+C_{o} \int_{0}^{b} \bar{F}(t) d t}{C_{m} \bar{F}(b)}
$$

$\mathrm{e}$

$$
\int_{b}^{b+T^{*}(b)} r(t) d t=T^{*}(b) r\left(b+T^{*}(b)\right)-\frac{C_{r}-C_{s}}{C_{m}}-\frac{C_{s}+C_{o} \int_{0}^{b} \bar{F}(t) d t}{C_{m} \bar{F}(b)}
$$


Substituindo a expressão acima na função de custo em (4.4), temos

$$
\begin{array}{r}
C\left(b, T^{*}(b)\right)=\frac{1}{T^{*}(b)}\left[\frac{C_{s}+C_{o} \int_{0}^{b} \bar{F}(t) d t}{\bar{F}(b)}-C_{s}\right]+\frac{C_{r}}{T^{*}(b)}+ \\
+\frac{C_{m}}{T^{*}(b)}\left[T^{*}(b) r\left(b+T^{*}(b)\right)-\frac{C_{r}-C_{m}}{C_{m}}-\frac{C_{s}+C_{o} \int_{0}^{b} \bar{F}(t) d t}{C_{m} \bar{F}(b)}\right] \\
=\frac{C_{s}+C_{o} \int_{0}^{b} \bar{F}(t) d t}{T^{*}(b) \bar{F}(b)}-\frac{C_{s}}{T^{*}(b)}+\frac{C_{r}}{T^{*}(b)}+C_{m} r\left(b+T^{*}(b)\right)-\frac{C_{r}-C_{s}}{T^{*}(b)}- \\
\frac{C_{s}+C_{o} \int_{0}^{b} \bar{F}(t) d t}{T^{*}(b) \bar{F}(b)},
\end{array}
$$

obtendo-se

$$
C\left(b, T^{*}(b)\right)=C_{m} r\left(b+T^{*}(b)\right) .
$$

Portanto, minimizar $C\left(b, T^{*}(b)\right)$ é equivalente a minimizar $r\left(b+T^{*}(b)\right)$ para $0 \leq b<\infty$. Como $r(t)$ é crescente em $\left[t_{2}, \infty\right)$ e $T^{*}(b)+b>t_{2}$, minimizar $C\left(b, T^{*}(b)\right)$ é equivalente a minimizar $\left(b+T^{*}(b)\right)$, como função de b.

Derivando a expressão (4.13) acima como função de b, temos as seguintes expressões equivalentes:

$$
\begin{aligned}
& T^{* *}(b) r\left(b+T^{*}(b)\right)+T^{*}(b) r^{\prime}\left(b+T^{*}(b)\right)\left(1+T^{* *}(b)\right)-r\left(b+T^{*}(b)\right)\left(1+T^{* *}(b)\right)+r(b)= \\
& \frac{1}{C_{m}}\left[\frac{C_{s} f(b)}{\bar{F}^{2}(b)}+\frac{C_{o} \bar{F}(b) \bar{F}(b)+f(b) C_{o} \int_{0}^{b} \bar{F}(t) d t}{\bar{F}^{2}(b)}\right] \Leftrightarrow \\
& \Leftrightarrow T^{\prime *}(b) r\left(b+T^{*}(b)\right)+T^{*}(b) r^{\prime}\left(b+T^{*}(b)\right)\left(1+T^{\prime *}(b)\right)-T^{\prime *}(b)\left(r\left(b+T^{*}(b)\right)\right)+r(b)= \\
& \frac{C_{o} \bar{F}^{2}(b)+f(b)\left(C_{s}+C_{o} \int_{0}^{b} \bar{F}(t) d t\right)}{C_{m} \bar{F}^{2}(b)} \Leftrightarrow \\
& \Leftrightarrow T^{\prime *}(b) r\left(b+T^{*}(b)\right)+T^{*}(b) r^{\prime}\left(b+T^{*}(b)\right)+T^{*}(b) T^{*}(b) r^{\prime}\left(b+T^{*}(b)\right)-r\left(b+T^{*}(b)\right)- \\
& -T^{\prime *}(b) r\left(b+T^{*}(b)\right)+r(b)=\frac{C_{o} \bar{F}^{2}(b)+f(b)\left(C_{s}+C_{o} \int_{0}^{b} \bar{F}(t) d t\right)}{C_{m} \bar{F}^{2}(b)} \Leftrightarrow
\end{aligned}
$$


$T^{*}(b) T^{*}(b) r^{\prime}\left(b+T^{*}(b)\right)=$

$$
=r\left(b+T^{*}(b)\right)-r(b)-T^{*}(b) r^{\prime}\left(b+T^{*}(b)\right)+\frac{C_{o} \bar{F}^{2}(b)+f(b)\left(C_{s}+C_{o} \int_{0}^{b} \bar{F}(t) d t\right)}{C_{m} \bar{F}^{2}(b)}
$$

Para $b \in\left[t_{1}, \infty\right)$ e $b+T^{*}(b)>t_{2}$, temos

$$
r\left(b+T^{*}(b)\right)-r(b)>0
$$

Consequentemente, de (4.16), concluímos que

$$
T^{*}(b) T^{*}(b) r^{\prime}\left(b+T^{*}(b)\right)>-T^{*}(b) r^{\prime}\left(b+T^{*}(b)\right)
$$

e que

$$
T^{*}(b)>-\frac{T^{*}(b) r^{\prime}\left(b+T^{*}(b)\right)}{T^{*}(b) r^{\prime}\left(b+T^{*}(b)\right)}=-1
$$

pois se $b+T^{*}(b)>t_{2}$ e dessa forma $r^{\prime}\left(b+T^{*}(b)\right)>0$. Portanto $\frac{\partial}{\partial b}\left(b+T^{*}(b)\right)>0, \forall b \geq t_{1}$ e, portanto, o valor mínimo de $b+T^{*}(b)$ só pode ser atingido no intervalo $\left[0, t_{1}\right]$, i é, $0 \leq b^{*} \leq t_{1}$ e $\left(b^{*}+T^{*}\left(b^{*}\right)\right)>t_{2}$.

Resumindo, vemos que no caso 1

$b+T^{*}(b)>t_{2}, \forall b \in B_{1}=[0, \infty)$ e $0 \leq b^{*} \leq t_{1}$, em que $b^{*}$ e $T^{*}(b)$ satisfazem

$$
\begin{aligned}
C\left(b^{*}, T^{*}\left(b^{*}\right)\right) & \leq C\left(b, T^{*}(b)\right), \forall b \neq b^{*} \text { e } b \in B_{1}=[0, \infty) \\
& <C(b, T), \forall T \neq T^{*}(b) \text { e } b \in B_{1}=[0, \infty) .
\end{aligned}
$$

Caso 2) $B_{1}=\emptyset$ e $B_{2}=[0, \infty)$

Como no caso 1 , podemos mostrar facilmente que para todo $b \geq 0$.

$\psi_{b}(T)<\frac{1}{C_{m} \bar{F}(b)}\left[\left(C_{r}-C_{m}\right) \bar{F}(b)+\left(C_{s}+C_{o} \int_{0}^{b} \bar{F}(t) d t\right)\right], \forall T \geq 0$ 
De (4.7) $\frac{\partial C}{\partial T}<0, \forall \quad b \geq 0$ e $T>0$ o que implica que

$C(b, T)>C(b, \infty) \forall b \geq 0, T>0$, i é, $T^{*}(b)=\infty \forall b \geq 0$.

Dessa forma,

$C\left(b, T^{*}(b)\right)=C(b, \infty)=C_{m} r(\infty), \forall b \geq 0$ e, portanto,

$C_{m} r(\infty)=C(b, \infty)<C(b, T), \forall b \geq 0 \forall T>0$

Caso 3) $B_{1} \neq \emptyset$ e $B_{2} \neq \emptyset$

Podemos notar que $\infty$ não está no fecho de $B_{1}$. Caso contrário, seja $\left\{b_{n}\right\}_{n \geq 1} \subseteq B_{1}$ tal que $b_{n}>t_{1}$, $\forall n \geq 1$ e $b_{n} \uparrow \infty$. Então de (4.10) teríamos

$\psi_{t_{1}}(\infty)>\psi_{b_{n}}(\infty)$

$$
>\frac{1}{C_{m} \bar{F}\left(b_{n}\right)}\left[\left(C_{r}-C_{m}\right) \bar{F}\left(b_{n}\right)+\left(C_{s}+C_{o} \int_{0}^{b_{n}} \bar{F}(t) d t\right)\right], \forall n \geq 1
$$

Fazendo $n \rightarrow \infty$ em (4.17), temos $\psi_{t_{1}}(\infty)=\infty$. Isto implica que $B_{1}=[0, \infty)$ e, assim, $B_{2}=\emptyset$, o que é uma contradição. Portanto, neste caso, $\infty$ deve pertencer ao fecho de $B_{2}$.

Desde que $\psi_{b}(\infty)$ é estritamente decrescente em $b \geq t_{1}$ e a função

$\frac{1}{C_{m} \bar{F}(b)}\left[\left(C_{r}-C_{m}\right) \bar{F}(b)+\left(C_{s}+C_{o} \int_{0}^{b} \bar{F}(t) d t\right)\right], \forall n \geq 1$

cresce estritamente para $\infty$, quando $b \uparrow \infty$, existe $s, 0 \leq s \leq \infty$ tal que $[s, \infty) \subseteq B_{2}$. Seja $\beta=\inf \left\{t:[t, \infty) \subseteq B_{2}\right\}$. Claramente $[\beta, \infty) \subseteq B_{2}$.

Primeiro, suponha $\beta \leq t_{1}$.

Desde que $B_{1}$ é um conjunto aberto, existe um intervalo aberto $\left(b_{1}, \beta\right) \subseteq B_{1}$. Como no caso 2, podemos mostrar que $T^{*}(b)=\infty, \forall b \in B_{2}$, e, então

$$
\min _{b \geq \beta} C\left(b, T^{*}(b)\right)=\min _{b \geq \beta} C(b, \infty)=C_{m} r(\infty)
$$


Contudo, para qualquer $\left[b^{\prime}, b^{\prime \prime}\right] \subseteq\left(b_{1}, \beta\right)$ podemos mostrar, como no caso 1 , que existe $\tilde{b} \in\left[b^{\prime}, b^{\prime \prime}\right]$ tal que

$$
\begin{aligned}
\min _{b_{1}<b<\beta} C\left(b, T^{*}(b)\right) & \leq \min _{b^{\prime} \leq b \leq b^{\prime \prime}} C\left(b, T^{*}(b)\right) \\
& =C_{m} r\left(\tilde{b}+T^{*}(\tilde{b})\right) \\
& <C_{m} r(\infty)
\end{aligned}
$$

Portanto, o tempo ótimo de burn-in, $b^{*}$, satisfaz $b^{*}<\tilde{b} \leq b^{\prime \prime}<\beta \leq t_{1}$.

Agora, supondo $\beta>t_{1}$, resulta que $\left[t_{1}, \beta\right) \subseteq B_{1}$. Contudo, $B_{1}$ é um conjunto aberto e existe um $b_{1} \in\left(0, t_{1}\right)$ tal que $\left(t_{1}, \beta\right) \subseteq B_{1}$. Por argumento semelhante ao caso $\beta \leq t_{1}$ concluímos, que $b^{*} \leq t_{1}$.

\section{Exemplo 4.1:}

Como exemplo de aplicação considere o Exemplo 3.1 do Capítulo 3. Então o tempo ótimo de burn-in $b^{*}$ e a política de manutenção ótima $T^{*}$ devem satisfazer a equação:

$$
\begin{aligned}
T\left(0.5 \lambda^{0.5}(b+T)^{0.5-1} e^{(\lambda(b+T))^{0.5}}\right) & -\int_{b}^{b+T} 0.5 \lambda^{0.5} t^{0.5-1} e^{(\lambda t)^{0.5}} d t= \\
& =\frac{1}{c_{m} e^{1-e^{(\lambda b)^{0.5}}}}\left[\left(c_{r}-c_{s}\right) e^{1-e^{(\lambda b)^{0.5}}}+c_{s}+c_{o} \int_{0}^{b} e^{1-e^{(\lambda t)^{0.5}}} d t\right]
\end{aligned}
$$

Os resultados da simulação para este caso também apresentaram o mesmo comportamento, como mostrado anteriormente, o que comprova o Teorema, ou seja, a solução deve estar em $b^{*} \leq t_{1}$ e $b^{*}+T^{*}>t_{2}$. 


\section{Capítulo 5}

\section{Modelos Gerais: Comentários}

\subsection{Burn-in com reparos mínimos e política de manutenção por blocos com reparos mínimos}

Cha (2000) propõe um novo procedimento de burn-in para um objeto reparável. Durante o procedimento de burn-in, realiza-se um reparo mínimo na falha do objeto e não mais um reparo completo, como no Capítulo 4. Devido às realizações dos reparos mínimos, este novo procedimento de burn-in se mostra mais econômico e eficiente.

No Capítulo anterior, consideramos um tempo de burn-in b, fixado, e colocamos o objeto em teste. Se o objeto falha antes do tempo b, substituimo-o a um custo $C_{s}>0 \mathrm{e}$, assim, sucessivamente até que o objeto sobreviva ao tempo b. Assumindo que o reparo é completo, como no Capítulo 4, e considerando uma política de manutenção por bloco com reparos mínimos, o custo médio total por 
unidade de tempo, assintótico, que denotaremos por $C_{1}(b, T)$, é

$$
C_{1}(b, T)=\frac{1}{T}\left[-C_{s}+\frac{C_{s}+C_{o} \int_{0}^{b} \bar{F}(t) d t}{\bar{F}(b)}\right]+\frac{C_{r}+C_{m} \int_{b}^{b+T} r(t) d t}{T}
$$

No Capítulo 4, demonstramos algumas propriedades do tempo ótimo de burn-in $b^{*}$ e da política ótima de manutenção $T^{*}$ sob a suposição de que a taxa de falha $r(t)$ tinha a forma bathtub. Este procedimento de burn-in é muito aplicado nas situações em que os objetos que falham são desconsiderados. Quando os objetos submetidos ao burn-in admitem reparos mínimos e são de alguma complexidade, a sua substituição pode ser economicamente inviável e um novo procedimento se faz necessário, conforme a proposta abaixo, por exemplo.

Consideramos um tempo de burn-in b, fixado, e colocamos o objeto em teste. Quando o objeto falha antes do tempo b, efetuamos um reparo mínimo a um custo $C_{s m}>0$, continuamos o procedimento com o objeto reparado e, assim, sucessivamente. Imediatamente após o tempo b o objeto é colocado em operação e uma política de manutenção por blocos com reparos mínimos é adotada.

A menos do reparo mínimo esse novo procedimento de burn-in é semelhante ao anterior, em que é realizado um reparo completo. Nota-se que o tempo total de burn-in é a constante b.

Duas parcelas compõem o custo deste novo procedimento de burn-in. A primeira é proporcional ao tempo total de burn-in com constante de proporcionalidade $C_{o}$ e a outra é proporcional ao número de reparos mínimos durante o processo de burn-in. Assim, o custo deste novo procedimento de burn-in é

$$
h(b)=C_{o} b+C_{s m} N_{m}(b),
$$

onde $N_{m}(b)$ é o número de reparos mínimos em $(0, \mathrm{~b}]$. O custo médio é

$$
k(b)=E[h(b)]=C_{o} b+C_{s m} \int_{0}^{b} r(t) d t
$$


e o custo médio por unidade de tempo, assintótico, é

$$
\lim _{t \rightarrow \infty} \frac{E\left[N_{r}\lfloor(t)\rfloor+1\right] E[h(b)]}{t}=\lim _{t \rightarrow \infty}\left[\frac{\frac{t}{T}}{t}+\frac{1}{t}\right]\left[C_{o} b+C_{s m} \Lambda(b)\right]
$$

isto é:

$$
\lim _{t \rightarrow \infty} \frac{E\left[N_{r}\lfloor(t)\rfloor+1\right] E[h(b)]}{t}=\frac{1}{T}\left[C_{o} b+C_{s m} \Lambda(b)\right]
$$

onde $\Lambda(b)=\int_{0}^{b} r(t) d t$.

Portanto, o custo total médio por unidade de tempo, assintótico, é

$$
C_{2}(b, T)=\frac{1}{T}\left[C_{o} b+C_{s m} \int_{0}^{b} r(t) d t\right]+\frac{C_{r}+C_{m} \int_{b}^{b+T} r(t) d t}{T}
$$

É fácil notar que

$$
\begin{aligned}
& C_{2}(0, T)=C_{1}(0, T) ; 0<T \leq \infty \\
& C_{2}(b, \infty)=C_{1}(b, \infty) ; 0 \leq b<\infty .
\end{aligned}
$$

No caso geral, o teorema seguinte compara os custos ótimos para os dois procedimentos:

\section{Teorema 5.1.1:}

Sejam $b_{i}^{*}$ o tempo ótimo de burn-in e $T_{i}^{*}$ a política de manutenção ótima, satisfazendo

$$
C_{i}\left(b_{i}^{*}, T_{i}^{*}\right)=\min _{b \geq 0 ; T>0} C_{i}(b, T), i=1,2
$$

Então, se $0<C_{s m}<C_{s}$ temos

$$
C_{2}\left(b_{2}^{*}, T_{2}^{*}\right) \leq C_{2}\left(b_{1}^{*}, T_{1}^{*}\right) \leq C_{1}\left(b_{1}^{*}, T_{1}^{*}\right)
$$

para todo $0 \leq b_{1}^{*}<\infty$ e $0<T_{1}^{*}<\infty$. 


\section{Prova:}

Observe que no procedimento anterior, o número médio de reparos completos é $E(\eta-1)=\frac{F(b)}{\bar{F}(b)}$, onde $\eta$ tem distribuição geométrica. Contudo,

$$
\begin{aligned}
\frac{F(b)}{\bar{F}(b)} & =\frac{1-\bar{F}(b)}{\bar{F}(b)}=\frac{1-e^{-\int_{0}^{b} r(t) d t}}{e^{-\int_{0}^{b} r(t) d t}}=\left(1-e^{-\int_{0}^{b} r(t) d t}\right) e^{\int_{0}^{b} r(t) d t} \\
& =e^{\int_{0}^{b} r(t) d t}-1>\int_{0}^{b} r(t) d t=\Lambda(b)=E\left[N_{m}(b)\right], \forall b>0
\end{aligned}
$$

onde na última desigualdade usamos o fato de que se $x>0$

$$
e^{x}-1=\int_{0}^{x} e^{s} d s>\int_{0}^{x} 1 d s=x
$$

Portanto,

$$
\frac{F(b)}{\bar{F}(b)}>\Lambda(b) \forall b>0
$$

isto é, o número médio de reparos completos é maior que o número médio de reparos mínimos.

Para comparar as equações (5.1) e (5.2), é suficiente analisar o custo médio do processo de burn-in, pois o custo de operação,

$$
\frac{C_{r}+C_{m} \int_{b}^{b+T} r(t) d t}{T}
$$

é comum nas duas equações.

Como $\Lambda(b)<\frac{F(b)}{\bar{F}(b)}, C_{s m}<C_{s}$ por hipótese, e $\int_{o}^{b} \bar{F}(t) d t \geq \bar{F}(b) b$, temos

$$
\begin{gathered}
-C_{s}+\frac{C_{s}+C_{o} \int_{o}^{b} \bar{F}(t) d t}{\bar{F}(b)}=\frac{C_{s} F(b)+C_{o} \int_{o}^{b} \bar{F}(t) d t}{\bar{F}(b)} \geq \\
\geq \frac{C_{s m} F(b)+C_{o} b \bar{F}(b)}{\bar{F}(b)}>C_{s m} \Lambda(b)+C_{o} b
\end{gathered}
$$


e concluímos que

$$
C_{2}(b, T)<C_{1}(b, T)
$$

para todo $0 \leq b<\infty$ e $0<T \leq \infty$. Consequentemente

$$
C_{2}\left(b_{2}^{*}, T_{2}^{*}\right)<C_{2}\left(b_{1}^{*}, T_{1}^{*}\right)<C_{1}\left(b_{1}^{*}, T_{1}^{*}\right)
$$

para todo $0 \leq b_{1}^{*}<\infty$ e $0<T_{1}^{*} \leq \infty$.

As propriedades de $b_{2}^{*}$ e $T_{2}^{*}$ na equação 5.3, são dadas no teorema abaixo.

\section{Teorema 5.1.2:}

Se a taxa de falha $r(t)$ tem forma bathtub, é diferenciável e se o conjunto

$$
B_{1}=\left\{b \geq 0: \int_{b}^{\infty}[r(\infty)-r(t)] d t>\frac{1}{C_{m}}\left[C_{r}+C_{o} b+C_{s m} \int_{0}^{b} r(t) d t\right]\right\}
$$

é diferente do vazio, então $0 \leq b_{2}^{*}<t_{1}$ e $\infty>b_{2}^{*}+T_{2}^{*}(b)>t_{2}$, onde $T_{2}^{*}(b)$ é a solução única da equação

$$
\operatorname{Tr}(b+T)-\int_{b}^{b+T} r(t) d t=\frac{1}{C_{m}}\left[C_{r}+C_{o} b+C_{s m} \int_{0}^{b} r(t) d t\right]
$$

e $b^{*}$ é o tempo ótimo de burn-in, isto é,

$$
C_{2}\left(b^{*}, T_{2}^{*}(b)\right)<C_{2}\left(b_{2}, T_{2}^{*}(b)\right)<C_{2}(b, T), \forall b \neq b^{*}, \quad b \geq 0 \text { e } T \neq T_{2}^{*}(b) .
$$

Caso contrário, se $B_{1}$ é vazio, então para qualquer $b \geq 0$, temos $T_{2}^{*}(b)=\infty \quad$ e $C_{2}\left(b, T_{2}^{*}(b)\right)=C_{m} r(\infty)$, onde $T_{2}^{*}(b)$ satisfaz

$$
C_{2}\left(b_{2}, T_{2}^{*}(b)\right)<C_{2}(b, T), \forall b \geq 0 \text { e } T \neq T_{2}^{*}(b) .
$$

A prova do teorema acima é análoga a do teorema demonstrado no Capítulo 4 e pode ser encontrada em Cha (2000). 


\subsection{Procedimento de burn-in condicionado a tipos de falhas do objeto}

Cha (2001) considera dois tipos de falhas para o objeto: a falha do tipo I que não é crítica e o objeto pode voltar a funcionar após um reparo mínimo ou completo. A falha do tipo II que é crítica e só admite um reparo completo.

Independentemente do tempo de vida do objeto, a falha do tipo II ocorre com probabilidade $p$, $0 \leq p \leq 1$, e a falha do tipo I com probabilidade $1-p$. Neste contexto, Cha (2001) considera dois procedimentos de burn-in:

No Procedimento I um objeto novo é submetido a um processo de burn-in com tempo determinado b (fixado). Se o objeto falha antes de b é reparado completamente a um custo $C_{s}$, independente do tipo de falha. O objeto reparado é submetido ao mesmo procedimento de burn-in e, assim, sucessivamente.

No Procedimento II um objeto novo é submetido a um processo de burn-in com tempo determinado b (fixado). Se o objeto tem uma falha do tipo I, antes de b, é minimamente reparado a um custo $C_{s m}, 0<C_{s m}<C_{s}$, e se a falha é do tipo II é reparado completamente a um custo $C_{s}$. O objeto reparado é submetido ao mesmo procedimento de burn-in e, assim, sucessivamente.

Observe que o Procedimento I termina quando não existir falha no intervalo $(0, b]$, enquanto que o Procedimento II termina quando não existir falha do tipo II em $(0, b]$.

Consideramos uma política de manutenção por idade $\mathrm{T}$ em objetos que sobreviveram ao procedimento de burn-in. Quando em operação, este objeto é substituído por um novo (que também sobreviveu ao procedimento de burn-in) no tempo $\mathrm{T}$ (fixado) ou no tempo da primeira falha do tipo II, o que ocorrer primeiro. Reparos mínimos são realizados quando a falha do objeto em operação for do tipo I.

Na prática, o Procedimento I é mais comum. Em geral, o Procedimento II não pode ser aplicado 
pois os reparos mínimos são impraticáveis ou economicamente inviáveis. Por outro lado, se os dois procedimentos podem ser aplicados resulta que, sob certas condições, o Procedimento II produz um custo médio menor do que o Procedimento I.

Seja $X$ o tempo de vida de um objeto com função de sobrevivência $\bar{F}(t)=P(X>t)$, $\bar{F}(t)>0, \forall t \in \Re$ e função densidade $f(t)$. A taxa de falha do objeto é dada por $r(t)$

$$
r(t)=\frac{f(t)}{\bar{F}(t)}=\lim _{d t \rightarrow 0} \frac{P(X \in(t, t+d t] \mid X \geq t)}{d t}
$$

Se $\mathrm{X}$ sobrevive a um tempo de burn-in b, o tempo de vida residual $X_{b}$ tem função de sobreviência $P\left(X_{b}>t\right)=\frac{\bar{F}(b+t)}{\bar{F}(b)}$ e taxa de falha $r_{X_{b}}(t)=r(b+t)$.

Sejam $Y_{b}$ o tempo da primeira falha do tipo II após o burn-in do objeto e $\bar{F}_{Y_{b}}(t)=P\left(Y_{b}>t\right)$ a sua função de sobrevivência. É fácil notar que

$$
\begin{aligned}
r_{Y_{b}}(t) & =\lim P\left(Y_{b} \in(t, t+d t] \mid Y_{b} \geq t\right)= \\
& =\lim P\left(X_{b} \in(t, t+d t], \text { falha do tipo } I I \mid X_{b} \geq t\right) \\
& =\lim P\left(X_{b} \in(t, t+d t] \mid X_{b} \geq t\right) P(\text { falha do tipo } I I) \\
& =r_{X_{b}}(t) p=r(b+t) p
\end{aligned}
$$

e, portanto,

$$
\begin{aligned}
\bar{F}_{Y_{b}}(t)=P\left(Y_{b}>t\right)= & \exp \left(-\int_{0}^{t} p r(b+u) d u\right)=\exp \left\{-p \int_{b}^{b+t} r(s) d s\right\}= \\
= & \exp (-p[\Lambda(b+t)-\Lambda(b)]),
\end{aligned}
$$

onde $\Lambda(t)=\int_{0}^{t} r(s) d s$. 
Se definirmos $Z_{b}=\min \left(Y_{b}, T\right)$, então seu valor esperado é

$$
\begin{aligned}
E\left[Z_{b}\right] & =E\left(Z_{b} \mid Y_{b} \leq T\right) P\left(Y_{b} \leq T\right)+E\left(Z_{b} \mid Y_{b}>T\right) P\left(Y_{b}>T\right) \\
& =\int_{0}^{T} t d F_{Y_{b}}(t)+T \bar{F}_{Y_{b}}(T) .
\end{aligned}
$$

Fazendo $u=t$ e $d v=d F_{Y_{b}}(t)$ temos

$$
\begin{aligned}
& =T F_{Y_{b}}(T)-\int_{0}^{T} F_{Y_{b}}(t) d t+T \bar{F}_{Y_{b}}(T) \\
& =T-T \bar{F}_{Y_{b}}(T)-\int_{0}^{T} F_{Y_{b}}(t) d t+T \bar{F}_{Y_{b}}(T) \\
& =\int_{0}^{T} 1 d t-\int_{0}^{T} F_{Y_{b}}(t) d t
\end{aligned}
$$

e, portanto,

$$
E\left[Z_{b}\right]=\int_{0}^{T} \bar{F}_{Y_{b}}(t) d t
$$

Se denotarmos por $N(b, T)$ o número total de reparos mínimos (de um objeto que sobreviveu ao procedimento de burn-in de tempo b) que ocorrem durante sua operação sob uma política de manutenção $\mathrm{T}$, temos

$$
\begin{aligned}
E[N(b, T)] & =E\left[N(b, T), Y_{b} \leq T\right]+E\left[N(b, T), Y_{b}>T\right]= \\
& =\int_{0}^{T} E\left[N(b, T) \mid Y_{b}=t\right] d F_{Y_{b}}(t)+\int_{0}^{T}(1-p) r(b+u) d u \bar{F}_{Y_{b}}(T), \\
& =\int_{0}^{T} \int_{0}^{t}(1-p) r(b+u) d u d F_{Y_{b}}(t)+\int_{0}^{T}(1-p) r(b+u) d u \bar{F}_{Y_{b}}(T) \\
& =\left(\frac{1}{p}-1\right)(1-\exp \{-p[\Lambda(b+T)-\Lambda(b)]\}) .
\end{aligned}
$$

Vamos considerar uma política de manutenção por idade para objetos que sobreviveram ao Procedimento I de burn-in. Um objeto em operação é substituído na idade T (fixado) ou na primeira 
falha do tipo II, o que ocorrer primeiro. Sejam $C_{f}$ o custo para cada falha do tipo II, $C_{a}, 0<C_{a}<$ $C_{f}$, o custo de cada substituição planejada na idade $T>0$ e $C_{m}$ o custo do reparo mínimo realizado durante a operação.

Como antes, definimos $\eta-1$ como a variável aleatória que conta o número de reparos completos até que o primeiro objeto sobreviva ao processo de burn-in. $\eta$ tem distribuição geométrica dada por

$$
P(\eta=k)=\bar{F}(b) F^{k-1}(b) ; \forall k \geq 1
$$

e o custo médio durante o processo de burn-in, $k_{f}(b)$, é

$$
k_{f}(b)=C_{o} \frac{\int_{0}^{b} \bar{F}(t) d t}{\bar{F}(b)}+C_{s} \frac{F(b)}{\bar{F}(b)}
$$

Então, segue de (5.4)-(5.6) e por argumentos similares aos descritos no Capítulo 4, que o custo médio assintótico, $C_{1}(b, T)$, é

$$
\frac{1}{\int_{0}^{T} \bar{F}_{Y_{b}}(t) d t}\left\{k_{f}(b)+C_{m}\left[\left(\frac{1}{p}-1\right)(1-\exp \{-p[\Lambda(b+T)-\Lambda(b)]\})\right]+C_{f} F_{Y_{b}}(T)+C_{a} \bar{F}_{Y_{b}}(T)\right\}(5.7)
$$

onde a expressão entre colchetes é o número médio de reparos do objeto em operação.

Observe que se $p=1$, este procedimento se resume ao do Capítulo 3 para política de manutenção por idade, e se $p=0$, o procedimento se resume ao do Capítulo 4 para a política de manutenção por bloco quando $C_{a}=C_{r}$. Portanto, assumimos que $0<p<1$.

Consideramos agora uma política de manutenção por idade para objetos que sobreviveram ao Procedimento II de burn-in. Um objeto é substituído na idade $\mathrm{T}$ ou na primeira falha do tipo II, a que ocorrer primeiro. Realizamos reparos mínimos nas falhas do tipo I.

Observe que neste caso a v.a. $\eta$ tem distribuição geométrica dada por

$$
P(\eta=k)=\bar{F}_{I I}(t) F_{I I}^{k-1}(t) ; \forall k \geq 1
$$


onde $\bar{F}_{I I}(t)=\exp \{-p \Lambda(t)\}, \forall t \geq 0$, é a função de de sobrevivência de uma falha do tipo II de um objeto novo e que o custo médio durante o processo de burn-in, $k_{F_{I I}}(b)$, é

$$
C_{o} \frac{\int_{0}^{b} \bar{F}_{I I}(t) d t}{\bar{F}_{I I}(b)}+\frac{F_{I I}(b)}{\bar{F}_{I I}(b)}\left[C_{s}+C_{s m} \frac{1}{F_{I I}(b)} \int_{0}^{b} \int_{0}^{t}(1-p) r(u) d u d F_{I I}(t)\right]+C_{s m}(1-p) \Lambda(b)
$$

onde $\frac{1}{\bar{F}_{I I}(b)} \int_{0}^{b} \int_{0}^{t}(1-p) d F_{I I}(t)$ é o número médio de reparos mínimos quando uma falha do tipo II ocorre no intervalo $(0, b]$ e $(1-p) \Lambda(b)$ é o número médio de reparos mínimos quando não ocorre falha do tipo II em $(0, b]$.

Equivalentemente,

$$
k_{F_{I I}}(b)=C_{o} \frac{\int_{0}^{b} \bar{F}_{I I}(t) d t}{\bar{F}_{I I}(b)}+C_{s} \frac{F_{I I}(b)}{\bar{F}_{I I}(b)}+C_{s m}\left(\frac{1}{p}-1\right)[\exp \{p \Lambda(b)\}-1] .
$$

Portanto o custo médio por unidade de tempo, assintótico, $C_{2}(b, T)$, é

$$
\frac{1}{\int_{0}^{T} \bar{F}_{Y_{b}}(t) d t}\left\{k_{F_{I I}}(b)+C_{m}\left[\left(\frac{1}{p}-1\right)(1-\exp \{-p[\Lambda(b+T)-\Lambda(b)]\})\right]+C_{f} F_{Y_{b}}(T)+C_{a} \bar{F}_{Y_{b}}(t)\right\}
$$

Observe que se $p=1$, este procedimento se resume ao do Capítulo 3 para política de manutenção por idade e se $p=0$, o procedimento se resume ao da Seção 5.1. Portanto, assumimos que $b \in(0,1)$.

A relação entre os dois procedimentos é dada pelo teorema abaixo:

\section{Teorema 5.2.1:}

Para os custos $C_{1}(b, T)$ e $C_{2}(b, T)$ vale o seguinte

i) $C_{2}(0, T ; p)=C_{1}(0, T ; p), \forall T \in(0, \infty], p \in(0,1)$

ii) $C_{2}(b, T ; p)<C_{1}(b, T ; p), \forall b \in(0, \infty), T \in(0, \infty], p \in(0,1)$ 


\section{Prova:}

A condição (i) é imediata e clara;

Para provar a condição (ii) vamos considerar dois casos:

1) Quando $0<b<\infty$ e $0<T<\infty$,

observe que $C_{2}(b, T ; p)<C_{1}(b, T ; p)$ se, e somente se,

$$
g(b, p)=\left[C_{1}(b, T ; p)-C_{2}(b, T ; p)\right] \int_{0}^{T} \bar{F}_{Y_{b}}(t) d t>0
$$

Contudo, das expressões para $C_{1}(b, T ; p)$ e $C_{2}(b, T ; p)$ temos que $g(b, p)$

$=C_{o} \frac{\int_{0}^{b} \bar{F}(t) d t}{\bar{F}(b)}+C_{s} \frac{F(b)}{\bar{F}(b)}-\left[C_{o} \frac{\int_{0}^{b} \bar{F}_{I I}(t) d t}{\bar{F}_{I I}(b)}+C_{s} \frac{F_{I I}(b)}{\bar{F}_{I I}(b)}+C_{s m}\left(\frac{1}{p}-1\right)[\exp \{p \Lambda(b)\}-1]\right]$

e consequentemente $g(b, p)$

$$
\begin{gathered}
=C_{o} \int_{0}^{b} \exp \{\Lambda(b)-\Lambda(t)\} d t+C_{s} \exp \{\Lambda(b)\}-\left[C_{o} \int_{0}^{b} \exp \{p[\Lambda(b)-\Lambda(t)]\} d t+C_{s} \exp \{p \Lambda(b)\}\right. \\
+C_{s m}\left(\frac{1}{p}-1\right)[\exp \{p \Lambda(b)\}-1]>C_{s}\left[\exp \{\Lambda(b)\}-\frac{1}{p} \exp \{p \Lambda(b)\}+\frac{1}{p}-1\right] .
\end{gathered}
$$

Para um $0<b<\infty$, seja

$$
\phi(p)=\left[\exp \{\Lambda(b)\}-\frac{1}{p} \exp \{p \Lambda(b)\}+\frac{1}{p}-1\right] .
$$

Note que

$$
\begin{aligned}
\lim _{p \rightarrow 0} \phi(p) & =\exp \{\Lambda(b)\}-1-\lim _{p \rightarrow 0} \frac{1}{p} \exp \{p \Lambda(b)-1\} \\
& =\exp \{\Lambda(b)\}-1-\lim _{p \rightarrow 0} \Lambda(b) \exp \{p \Lambda(b)\}=\exp \{\Lambda(b)\}-1-\Lambda(b)
\end{aligned}
$$


e que

$$
\lim _{p \rightarrow 1} \phi(p)=0, \forall b>0
$$

A derivada de $\phi(p)$ em relação a $p$ é dada por

$$
\phi^{\prime}(p)=\frac{1}{p^{2}} \exp \{p \Lambda(b)\}[1-p \Lambda(b)-\exp \{-p \Lambda(b)\}]
$$

Usando o fato de que, $e^{x}-1>x, \forall x>0$, já mostrado anteriormente, temos que $\phi^{\prime}(p)<0$. Como $\lim _{p \rightarrow 1} \phi(p)=0, \lim _{p \rightarrow 0} \phi(p)>0$ e $\phi(p)$ é decrescente para $0<p<1$, concluímos que $\phi(p)>0$ e, portanto, $g(b, p)>0$ para todo $0<b<\infty$ e $0<p<1$.

2) Quando $0<b<\infty$ e $T=\infty$

Por argumentos descritos anteriormente, é fácil notar que

$$
C_{2}(b, \infty, p)<C_{1}(b, \infty, p), \forall 0<b<\infty, 0<p<1
$$

o que prova o Teorema.

Quando a probabilidade da falha do tipo II é igual a 1, o Procedimento II reduz-se ao Procedimento I. Assim, o custo total médio por unidade de tempo, assintótico, para os dois procedimentos pode ser colocado na forma $C_{1}(b, T)=$

$$
=\frac{1}{\int_{0}^{T} \bar{F}_{Y_{b}}(t) d t}\left\{k(b)+C_{m}\left[\left(\frac{1}{p}-1\right)(1-\exp \{-p[\Lambda(b+T)-\Lambda(b)]\})\right]+C_{f} F_{Y_{b}}(T)+C_{a} \bar{F}_{Y_{b}}(T)\right\},
$$

onde $k(b)$ é o custo médio do processo de burn-in, para o procedimento II e dado por

$$
k(b)=C_{o} \int_{0}^{b} \exp \{-p[\Lambda(t)-\Lambda(b)]\} d t+C_{s}[\exp \{\Lambda(b)\}-1]+C_{s m}\left(\frac{1}{p}-1\right)[\exp \{p \Lambda(b)\}-1]
$$


e o custo médio do processo de burn-in para o procedimento I é dado por

$$
k(b)=C_{o} \int_{0}^{b} \exp \{-[\Lambda(t)-\Lambda(b)]\} d t+C_{s}[\exp \{\Lambda(b)\}-1]
$$

O tempo ótimo de burn-in $b^{*}$ e a política de manutenção ótima $T^{*}$ devem satisfazer

$$
C\left(b^{*}, T^{*}\right)=\min _{b \geq 0, T>0} C(b, T)
$$

De maneira semelhante aos resultados anteriores, as propriedades de $b^{*}$ e $T^{*}$ são afirmadas no teorema seguinte:

\section{Teorema 5.2.2:}

Se a taxa de falha $r(t)$ tem a forma bathtub é diferenciável e se o conjunto $B_{1}=\left\{b \geq 0: p r(\infty) \int_{b}^{\infty} \exp \{p[\Lambda(t)-\Lambda(b)]\} d t-1>\frac{1}{C_{m}\left(\frac{1}{p}-1\right)+\left(C_{f}-C_{a}\right)}\left(C_{a}+k(b)\right)\right\}$

é diferente do vazio, então $0 \leq b^{*} \leq t_{1}$ e $b^{*}+T^{*}\left(b^{*}\right)>t_{2}$, onde $T^{*}(b)$ é a solução única da equação $p r(b+T) \int_{b}^{b+T} \exp \{-p[\Lambda(t)-\Lambda(b)]\} d t+\exp \{-p[\Lambda(b+T)-\Lambda(b)]\}-1=\frac{C_{a}+k(b)}{C_{m}\left(\frac{1}{p}-1\right)+\left(C_{f}-C_{a}\right)}$ se $b^{*} \in B_{1}=[0, \infty)$ e $T^{*}\left(b^{*}\right)=\infty$ se $b \in B_{2}=[0, \infty) \backslash B_{1}$.

O tempo ótimo de burn-in $b^{*}$ é o valor que satisfaz

$$
C\left(b^{*}, T^{*}\left(b^{*}\right)\right)<\min _{0 \leq b \leq t_{1}} C\left(b, T^{*}(b)\right) .
$$

Se o conjunto acima é vazio, então $\left(b^{*}, T^{*}\right)=\left(b^{*}, \infty\right)$, com $0 \leq b^{*} \leq t_{1}$ e $b^{*}+T^{*}\left(b^{*}\right)>t_{2}$, e o tempo ótimo de burn-in $b^{*}$ é o valor que satisfaz

$$
C\left(b^{*}, \infty\right)<\min _{0 \leq b \leq t_{1}} C(b, \infty) .
$$


A prova é semelhante ao Teorema (4.1) do Capítulo 4 e pode ser encontrada em Cha (2001).

Cha (2003) generaliza os resultados anteriores para o caso em que a probabilidade de ocorrência de uma falha do tipo II é $p(t), 0 \leq p(t) \leq 1$, dependente do tempo. A falha do tipo I ocorre com probabilidade $1-p(t)$. Os dois procedimentos de burn-in, I e II, são considerados.

O custo médio por unidade de tempo, assintótico, para o procedimento I é dado por

$$
\begin{array}{r}
C_{1}(b, T, p(\cdot))=\frac{1}{\int_{0}^{T} \bar{F}_{Y_{b}}(t) d t}\left\{\left[C_{o} \int_{0}^{b} \exp \{-[\Lambda(t)-\Lambda(b)]\} d t+C_{s}[\exp \{\Lambda(b)\}-1]\right]+\right. \\
\left.+C_{m}\left[\int_{0}^{T} r(b+t) \bar{F}_{Y_{b}}(t) d t-F_{Y_{b}}(T)\right]+C_{f} F_{Y_{b}}(T)+C_{a} \bar{F}_{Y_{b}}(t)\right\}
\end{array}
$$

onde $\Lambda(t)=\int_{0}^{t} r(u) d t$ e $\bar{F}_{Y_{b}}(t)=\exp \left\{\int_{b}^{b+T} p(u) r(u) d u\right\}$.

O custo médio por unidade de tempo, assintótico, para o procedimento II é dado por

$$
\begin{array}{r}
C_{2}(b, T, p(\cdot))=\frac{1}{\int_{0}^{T} \bar{F}_{Y_{b}}(t) d t}\left\{\left[C_{o} \int_{0}^{b} \exp \left\{-\left[\Lambda_{p}(t)-\Lambda_{p}(b)\right]\right\} d t+C_{s}\left[\exp \left\{\Lambda_{p}(b)\right\}-1\right]+\right.\right. \\
\left.+C_{s m} \int_{0}^{b}(1-p(\cdot)) r(t) \exp \left\{-\left[\Lambda_{p}(t)-\Lambda_{p}(b)\right]\right\} d t\right]+ \\
\left.+C_{m}\left[\int_{0}^{T} r(b+t) \bar{F}_{Y_{b}}(t) d t-F_{Y_{b}}(T)\right]+C_{f} F_{Y_{b}}(T)+C_{a} \bar{F}_{Y_{b}}(T)\right\},
\end{array}
$$

onde $\Lambda_{p}(t)=\int_{0}^{t} r_{p}(u) d t$.

Pode-se provar então que, sob a hipótese de que $0<C_{s m} \leq C_{s}$,

$C_{2}(0, T ; p(\cdot))=C_{1}(0, T ; p(\cdot))$ para $0<T \leq \infty$,

$C_{2}(b, T ; p(\cdot)) \leq C_{1}(b, T ; p(\cdot))$ para $0<b<\infty, 0<T \leq \infty$,

para $0 \leq p(\cdot) \leq 1 \forall t \geq 0$ e $p(t) \neq p$ q.c. $0 \leq p \leq 1$.

Podemos concluir que, se os dois procedimentos são aplicáveis, o Procedimento II é preferível ao Procedimento I. 
Em adição, sob a hipótese de que a taxa de falha $r(t)$ tem a forma bathtub e a probabilidade de falha do tipo II, $p(t)$, também tem a forma bathtub, com $C_{f}-C_{a}>C_{m}$, as propriedades de que o tempo ótimo de burn-in $b_{i}^{*}, i=1,2$ é menor do que o máximo dos primeiros pontos de troca (de $r(t)$ e $p(t)$ ) e da existência da política de manutenção ótima $T^{*}$ são provadas em Cha (2003). 


\section{Conclusão}

Os resultados alcançados analiticamente nos Capítulos 3 e 4 são interessantes e produzem um limite superior para o tempo ótimo de burn-in $b^{*}$ e um limite inferior para a política de manutenção ótima $T^{*}(b)$.

É intuitivo que devemos colocar o objeto em operação depois do tempo ótimo de burn-in $b^{*}$, pois sua taxa de falha atualizada é truncada à esquerda em $b^{*}$, o que produz um objeto em melhores condições de uso, com taxa de falha menor e, talvez, próxima da taxa de falha constante (da vida útil do objeto).

Podemos interpretar, também, que, descontado o tempo de burn-in $b^{*}$, as substituições planejadas devam ser realizadas próximas do período de desgaste do objeto.

Um tópico especialmente importante que aparece no Capítuolo 5 é a comparação dos custos de políticas de manutenção para os procedimentos de burn-in diferentes. É intuitivo que um procedimento de burn-in que utiliza reparos mínimos é mais conveniente, pois produz um custo menor.

Duas questões se impõe como futuro objetos de estudos:

- Caracterização de classes de distribuições cujas misturas produzem distribuições adequadas para um procedimento de burn-in (por exemplo, taxa de falha na forma bathtub);

- Estimar os pontos de mudança de uma taxa de falha (por exemplo, $t_{1}$ e $t_{2}$ da taxa de falha na forma bathtub). 


\section{Parte I}

Apêndice A 
Simulação do Teorema 3.1 do Capítulo 3. Código em $\mathbb{R}$

$$
\begin{aligned}
& a=0.5 \\
& k=3 \\
& c o=1 \\
& c f=2 \\
& c a=1 \\
& j=0.3 / 20 \\
& \text { for }(b \text { in } \operatorname{seq}(\text { from }=j, \text { to }=.3, \text { length }=20))\{ \\
& \text { for }(t \text { in } \operatorname{seq}(\text { from }=(.3-j) / 100, \text { to }=.3-j, \text { length }=100))\{ \\
& e q 0<-\exp \left(1-\exp (k * b)^{a}\right) \\
& \left.e q 1=a *\left(k^{a}\right) *\left((b+t)^{(} a-1\right)\right) * \exp (k *(b+t))^{a} \\
& \text { int } 1=\text { function }(x) \exp \left(1-\left(\exp (k *(b+x))^{a}\right)\right) \\
& c p 1=\text { integrate }(\text { int } 1,0, t) \\
& e q 2=c p 1 \$ \text { value } / \text { eq } 0 \\
& e q 3=\left(\exp \left(1-\exp (k *(b+t))^{a}\right)\right) / e q 0 \\
& \text { int } 2=\text { function }(x) \exp \left(1-\left(\exp (k * x)^{a}\right)\right) \\
& c p 2=\text { integrate }(\text { int } 2,0, b) \\
& e q 4=(c o * c p 2 \$ \text { value })+(c s *(1-e q 0)) \\
& e q 5=e q 4 / e q 0 \\
& e q 6=(c f+e q 5) /(c f-c a) \\
& e q g=e q 1 *(e q 2)+e q 3-e q 6 \\
& \operatorname{print}(e q g) \\
& \} \\
& \text { \} }
\end{aligned}
$$


Simulação do Teorema 4.1 do Capítulo 4. Código em $\mathbb{R}$

$$
\begin{aligned}
& a=0.5 \\
& k=3 \\
& c o=1 \\
& c r=2 \\
& \mathrm{~cm}=1 \\
& c a=1 \\
& j=0.3 / 20 \\
& \text { for }(b \text { in } \operatorname{seq}(\text { from }=j, \text { to }=.3, \text { length }=20))\{ \\
& \text { for }(t \text { in } \operatorname{seq}(\text { from }=(.3-j) / 100, \text { to }=.3-j, \text { length }=100))\{ \\
& \operatorname{eq0}=\exp \left(1-\exp (k * b)^{a}\right) \\
& \left.e q 1=t *\left(a *\left(k^{a}\right) *\left((b+t)^{(} a-1\right)\right) * \exp (k *(b+t))^{a}\right) \\
& \text { int } \left.1=\text { function }(x) a *\left(k^{a}\right) *\left(x^{(} a-1\right)\right) *\left(\exp (k * x)^{a}\right) \\
& c p 1=\text { integrate }(\text { int } 1, b, t) \\
& \text { eq2 }=\text { cp } 1 \$ \text { value } \\
& e q 3=\left(\exp \left(1-\exp (k *(b+t))^{a}\right)\right) / e q 0 \\
& \text { int } 2=\operatorname{function}(x) \exp \left(1-\left(\exp (k * x)^{a}\right)\right) \\
& e q 3=1 /(c m * e q 0) \\
& e q 4=(c r-c s) * e q 0 \\
& \text { int } 2=\text { function }(x) \exp \left(1-\exp (k * x)^{a}\right) \\
& c p 2=\text { integrate }(\text { int } 2,0, b) \\
& e q 5=c s+(c o * c p 2 \$ v a l u e) \\
& e q g=e q 1-e q 2-(e q 3 *(e q 4+e q 5)) \\
& \text { print (eqg) } \\
& \text { \} } \\
& \text { \} }
\end{aligned}
$$




\section{Bibliografia}

[1] Barlow, R. E. and Proschan, F. (1965) Mathematical Theory of Reliability, John Wiley \& Sons, NY

[2] Block, H. W. and Savits, T.H. (1997) Burn-in, Statistical Science.Vol 12.No.1,1-19

[3] Cha, J. H. (2000) On a better burn-in procedure. J. Appl. Prob. 37, 1090-1103

[4] Cha, J. H. (2001) Burn-in procedure for a generalized model. J. Appl. Prob. 38, 542-553

[5] Cha, J. H. (2003) A further extension of the generalized Burn-in model. J. Appl. Prob. 40, 264-270

[6] Jensen, F. and Petersen, N. E. (1982) Burn-in. Wiley, New York

[7] Mi, J. (1991) Optimal burn-in. Doctoral Thesis, Departament of Statistics, University of Pittsburgh.

[8] Mi, J. (1994) Burn-in and maintenance polices. Advance Appl. Prob. 26,207-221 